\title{
Nonlinear Piecewise Restoring Force in Hydrokinetic Power Conversion using Flow Induced Motions of Single Cylinder
}

Chunhui Ma ${ }^{1,2,{ }^{*}}$, Hai Sun ${ }^{1,3,6, *}$, Gary Nowakowski ${ }^{4}$, Erik Mauer ${ }^{5}$, Michael M. Bernitsas ${ }^{1,7,8}$

1. Marine Renewable Energy Laboratory, Dept. of Naval Architecture \& Marine Engineering, University of Michigan, 2600 Draper Road, Ann Arbor, MI 48109-2145

2. Asst. Professor, Jiangsu Maritime Institute; Research Fellow MRELab, University of Michigan, Ann Arbor, Michigan, USA

3. Asst. Professor, Harbin Engineering University; Post-doctoral Fellow MRELab, University of Michigan, Ann Arbor, Michigan, USA

4. U.S. Department of Energy, Wind and Water Power Technologies Office, Golden Field Office

5. Allegheny Science \& Technology, Contractor to US-DOE

6. Corresponding Author: Phone: 734-353-3246, E-mail: sunhai2009@gmail.com

7. Department of Mechanical Engineering, University of Michigan

8. Vortex Hydro Energy, Ann Arbor, Michigan

* Equally Credited Authors

Submitted for Publication to:

OCEAN ENGINEERING

Initial submission: April 2016

Revision: 


\begin{abstract}
Flow Induced Motions (FIMs) of a single, rigid, circular cylinder with piecewise continuous restoring force are investigated for Reynolds number $24,000 \leq R e \leq 120,000$ with damping, and different piecewise functions as parameters. Selective roughness is applied to enhance FIM and increase the hydrokinetic energy captured by the VIVACE (Vortex Induced Vibration for Aquatic Clean Energy) Converter at higher Reynolds numbers. The second generation of virtual spring-damping system Vck, developed in the Marine Renewable Energy Laboratory (MRELab), enables embedded computer-controlled change of viscous-damping and spring-stiffness for fast and precise oscillator modeling. Experimental results for amplitude response, frequency response, energy harvesting, and efficiency are presented and discussed. All experiments were conducted in the Low Turbulence Free Surface Water (LTFSW) Channel of the MRELab of the University of Michigan. The main conclusions are: (1) The nonlinear piecewise spring Converter can harness energy from flows as slow as $0.275 \mathrm{~m} / \mathrm{s}$ with no upper limit. (2) In galloping, the nonlinear spring Converter has up to $76 \%$ better performance than its linear spring counterpart . (3) The FIM response is predominantly periodic for all nonlinear spring functions used. (4) Optimal power harnessing is achieved by changing the nonlinear piecewise spring function and the linear viscous damping. (5) VIVACE exhibits local maxima in power conversion at the end of the upper branch in VIV and the highest velocity reached in galloping. (6) The efficiency optima though are at the beginning of the VIV initial branch and at the beginning of galloping.
\end{abstract}

KEY WORDS: hydrokinetic energy; nonlinear restoring force; flow-induced motions; vortex-induced vibrations; galloping; distributed surface roughness 


\section{INTRODUCTION}

Background information on Flow Induced Motion (FIM), alternating lift in harnessing horizontal hydrokinetic energy, nonlinear restoring forces in oscillators, and the research objectives of this study are presented in the Introduction.

\subsection{Flow Induced Motions}

Flow Induced Motions (FIMs) present a source of challenge for diverse structures in steady flows such as heat exchangers, bridges, buildings, offshore structures, cables, pipelines, or power-transmission cables. The most common FIM phenomenon is Vortex-Induced Vibration (VIV), which was first observed by Leonardo da Vinci in 1504. The first mathematical formulation was done by Strouhal in 1878. Comprehensive reviews of VIV have been published by Bearman (1984, 2011), Sarpkaya (2004), and Williamson and Govardhan (2004). Galloping is another form of flow induced motion. It is an aero/hydro-elastic instability characterized by lower frequencies and larger amplitudes than VIV and is perpendicular to the flow (Blevins 1990). It is more vigorous and destructive than VIV, yet not as complex a phenomenon as VIV. Galloping occurs above a critical flow speed with no upper limit till structural failure. The driving mechanism is an instability related to negative damping caused by asymmetry in the relative flow. The latter can be due to a geometric asymmetry or a flow asymmetry due to an upstream wake. Thus, a smooth circular cylinder in a uniform flow cannot gallop.

Typically, as flow velocity increases, VIV appears first, followed by galloping. Figure 1 shows the various branches of FIM for a single cylinder with distributed surface roughness to 
induce galloping. The cylinder is rigid on elastic supports in a cross-flow. As velocity increases, FIM is initiated as VIV due to vortex shedding, alternating from the two sides of the cylinder, resulting in transverse movement of the cylinder. Vortex shedding locks onto the cylinder oscillation over a broad range of synchronization starting with the initial branch as shown in Figures 1 and 2. This response amplitude increases with the velocity of the flow in the upper branch. It is followed by the lower branch with smaller response, then the desynchronization zone, and the end of the VIV synchronization range. The various branch characteristics may differ significantly depending on whether the flow in flow regime TrSL2 or TrSL3 (Zdravkovitz, 1997), where TrSL indicates “Transition in Shear Layer". Williamson and Govardhan (2004) conducted experiments for Re about 3,800 in TrSL2 $(1,000<\operatorname{Re}<20,000)$ and response amplitude reached one cylinder diameter. Bernitsas et al. (2009) and Raghavan and Bernitsas (2010) conducted experiments for $16,000<R e<140,000$ in the TrSL3 $(20,000<R e<300,000)$ flow regime. They showed that: (a) the dependence of VIV on Reynolds number is strong; (b) in the upper branch in TrSL3, the amplitude increases reaching 1.8 diameters; and (c) the upper branch overtakes almost completely the lower branch. Figure 1 shows all the VIV branches. The spring-stiffness value has been selected low to initiate and end VIV early. The damping value has been selected high to initiate galloping late. That way, the full range of VIV synchronization is shown as VIV ends and the amplitude of oscillation nearly reaches zero before the onset of galloping, the gap between VIV and Galloping increases as the stiffness decreases, decreases as 
the total damping decreases The relative positioning of VIV vs. galloping is explained in detail by Park et al. (2013).

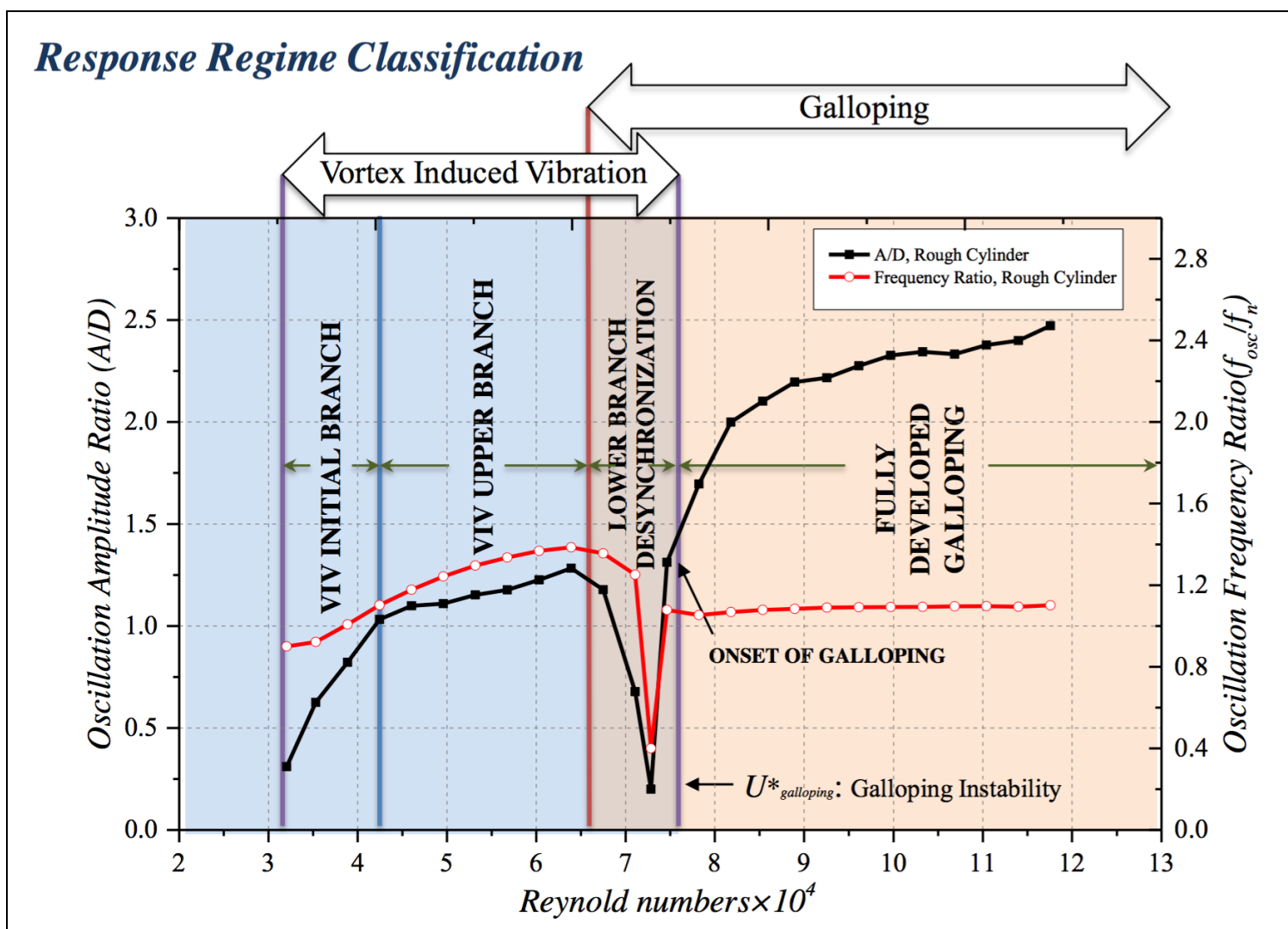

Figure 1. Flow Induced Motion regions with small overlap between Vortex Induced Vibrations and galloping; $K=400 \mathrm{~N} / \mathrm{m}, \quad \zeta=0.12$ (Sun et.al. 2016) 


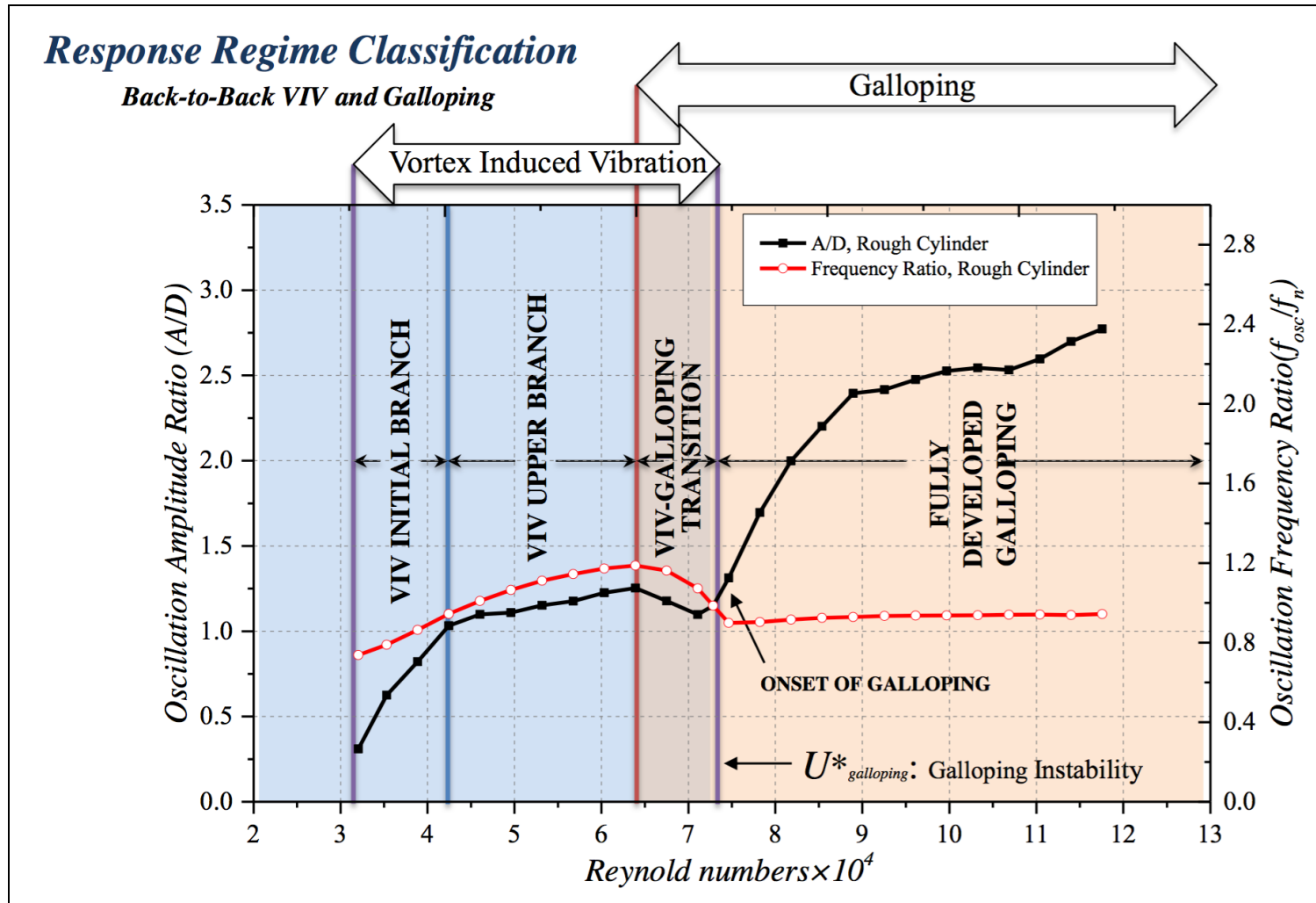

Figure 2. Flow Induced Motion regions with overlap of Vortex Induced Vibrations and galloping; $K=600 \mathrm{~N} /, \quad \zeta=0.04$ (Sun et.al. 2016)

\subsection{Alternating Lift in Hydrokinetic Energy}

FIMs are suppressed in engineering applications because of their destructive nature. By enhancing and controlling FIM, the Marine Renewable Energy Laboratory (MRELab) at the University of Michigan developed the VIVACE Converter (Bernitsas and Raghavan 2009, 2011, 2014; Bernitsas et al. 2008, 2009) to convert kinetic energy of water into electricity. This concept is being advanced continually in the MRELab through simulation, analysis, and scaled physical testing. Relevant research on harvesting MHK energy using vortex-induced vibrations has been published in several countries: Nishi, 2013 (Japan), Zhu, 2012 (USA), Meliga et al, 
2011 (Switzerland and France), Barrero-Gil, G. et al, 2012 (Spain), Konstantinidis, E., 2013

(Greece), Kang and Jia, 2013, (China).

Among alternating-lift technologies, the VIVACE converter is probably the closest to commercialization because it has undergone extensive laboratory testing and several field deployments since its introduction in 2006. The research objective of the Marine Renewable Energy Lab (MRELab) is to study FIM of single or multiple cylinders on springs and find ways to enhance FIM in order to design VIVACE Converters and optimize their power output for a broad range of flow velocities. Power envelopes as function of flow velocity and type of FIM need to be generated. FIMs studied in the process include vortex-induced vibrations, galloping, and gap flows. Generating power envelopes in such a large parametric design space and for different FIM phenomena is equivalent to finding the efficiency of propellers with different number of blades and geometric properties. Further, in open-water propeller tests all blades are in equivalent position while in schools of cylinders the hydrodynamics of each cylinder changes depending on the cylinder relative location in the school.

\subsection{Nonlinear Restoring Force in Oscillators}

Research has shown that fish move effortlessly in stationary or flowing water, in schools or individually, by generating alternating lift (Bernitsas, 2016). Fish utilize the flow velocity and achieve forward thrust passively or actively using powered movements by self-correcting mechanisms (J. Liao, 2007). Just like fish, VIVACE utilizes alternating lift generated in FIM to 
convert hydrokinetic energy to mechanical energy in the oscillating cylinders and subsequently electrical energy through a power-take-off system.

The driving mechanics are different in VIV and galloping even though both are due to alternating lift. VIV is driven by alternating vortex shedding due to lock-in over the entire range of VIV. Galloping is due to instability of the shear layer favored by the cylinder motion. When the cylinder motion is reversed the other shear layer drives the cylinder. Galloping has no upper-end in velocity until structural failure. In an infinitely wide apparatus, galloping would not require a spring restoring force. The cylinder would be driven in one direction by the instability on one side of the cylinder. In a real Converter where the width of the cylinder frame is finite, as in our experiments, the springs are needed to reverse the cylinder at the end of the travel for continuous energy conversion. The practical limitation of course is due to the experimental facility in which the width of the Converter frame has to fit. Thus, the response amplitude operator of the Converter can be designed as in Figure 2, so that it has high response from the initiation of VIV and on with out end. This is a major advantage compared to a linear resonator that has a narrow bandwidth of high response near its natural frequency (Bernitsas, 2016). Accordingly, a VIVACE Converter is designed with: (1) a low spring stiffness for low-speed VIV initiation, and (2) a low initial damping and strong geometric asymmetry for early galloping initiation (Blevins, 1990). Experimental results, like those in Figure 2, show that these design aspects can be achieved via different linear spring stiffness and damping. The following equations clarify these two design aspects. 
For mass ratio $m^{*}=m_{o s d} m_{d}$ on the order of 1 , the VIV synchronization range in terms of the reduced velocity $U^{*}$ is approximately

$$
\sim 5 U^{*}=\frac{U}{f_{n, \text { water }} D}<\sim 10
$$

where, $m_{o s c}$ is the oscillating mass, $m_{d}$ is the displaced fluid mass;

$$
f_{n, \text { water }}=\frac{1}{2 \pi} \sqrt{\frac{K}{m_{o s c}+m_{a}}}
$$

is the natural frequency in water, $m_{a}$ is the added mass, and $K$ is the total spring stiffness. That is, the end of the VIV synchronization range increases proportionally to the square root of the spring stiffness $K$.

On the other hand, galloping initiates at an absolute value of velocity $U=U_{\text {critical }}$ given by Blevins (1990) as

$$
U_{\text {cirtical }}=\frac{2 c_{\text {total }}}{\rho D \frac{\partial c_{y}}{\partial \alpha}}
$$

where $c_{\text {total }}$ is the linear viscous damping, $D$ is the cylinder diameter, and $\partial c_{y} / \partial \alpha$ is the derivative of the lift coefficient with respect to the angle of attack. $U_{\text {critical }}$ is not affected by the spring constant $K$ or the mass $\mathrm{m}$. That is, initiation of galloping does not depend on $K$ but only on the absolute flow velocity, system damping, and geometric particulars of the oscillator.

Comparing inequality (1) and equation (3), it is observed that the gap between VIV and galloping can be bridged by increasing $K$, decreasing $m$, decreasing $c_{\text {total }}$, or increasing $D$. Thus, 
an oscillator responding with high amplitude at a range of velocities staring at $U^{*} \simeq 5$ and having no upper end can be designed. That is, when it comes to energy capturing, the oscillators in the Converter provide a great advantage compared to linear resonators, which have high response within a very limited bandwidth in the vicinity of their natural frequency.

In spite of the fact that $U_{\text {critical }}$ is independent of $K$, the latter has an impact on galloping for energy harnessing as it affects the cylinder velocity and direction reversal point. It is thus, reasonable to expect that nonlinear, piecewise-continuous, stiffness may be a valid and possibly better alternative to a constant spring constant $K$ characteristic of a linear oscillator. Nonlinear restoring force has been studied in the past, however, no studies were related to power harnessing, or FIM, or enhancing FIM under high damping. Barton, et.al. (2009) studied an arrangement of magnets in conjunction with an iron-cored stator for broadly resonant response. Williamson et.al. (2013) studied cubic and quantic restoring force in VIV to measure the fluid structure interaction response; they also extended the VIV resonant response to a broader regime $(1,000<\operatorname{Re}<5,000)$.

\subsection{Research Objectives}

The objective of this paper is to systemically study the effects of piecewise continuous spring stiffness, with linear viscous damping in each piece, on a single cylinder VIVACE converter to: (a) Assess the cylinder FIM with nonlinear restoring spring stiffness; (b) Attain higher conversion of hydrokinetic energy to mechanical. Tests are conducted in the range of 24,000 $<\operatorname{Re}<120,000$, which falls in the high-lift TrSL3 flow regime. TrSL stands for Transition in Shear Layer and "3" indicates the third region where the shear layer is fully saturated resulting 
in stronger vortices, shorter formation length, and highest lift (Zdravkovitz, 1997). The physical model and running parameters are presented in Section 2. In Section 3, the mathematical model for the harnessed power is summarized. In Section 4, experimental results for amplitude response, frequency response, energy harvesting, and efficiency are presented and discussed. Conclusions are drawn at the end.

\section{PHYSICAL MODEL}

The experimental facility, the model oscillator, the virtual oscillator simulator, and the nonlinear spring models are described in this section.

\subsection{Experimental Facility LTFSW Channel}

All tests were conducted in the Low Turbulence Free Surface Water (LTFSW) Channel, in the MRELab of the University of Michigan (Fig. 3). The LTFSW Channel recirculates 10,000 gallons of fresh water at speed up to $1.4 \mathrm{~m} / \mathrm{s}$ using an impeller powered by a $20 \mathrm{hp}$ induction motor. The turbulence intensity normalized by the free-stream velocity was reported at $0.095 \%$ (Walker et al. 1996). The test section is $2.44 \mathrm{~m}$ long and $1.0 \mathrm{~m}$ wide, and is made of transparent plexi-glass, which enables visualization of the flow past cylinders using two $5 \mathrm{~W}$ argon lasers and aluminum oxide particles of $100 \mu \mathrm{m}$. For this study, the water depth at the test-section was set at $1.17 \mathrm{~m}$ and the maximum flow velocity was limited to $1.35 \mathrm{~m} / \mathrm{s}$ for safety against the galloping instability. 


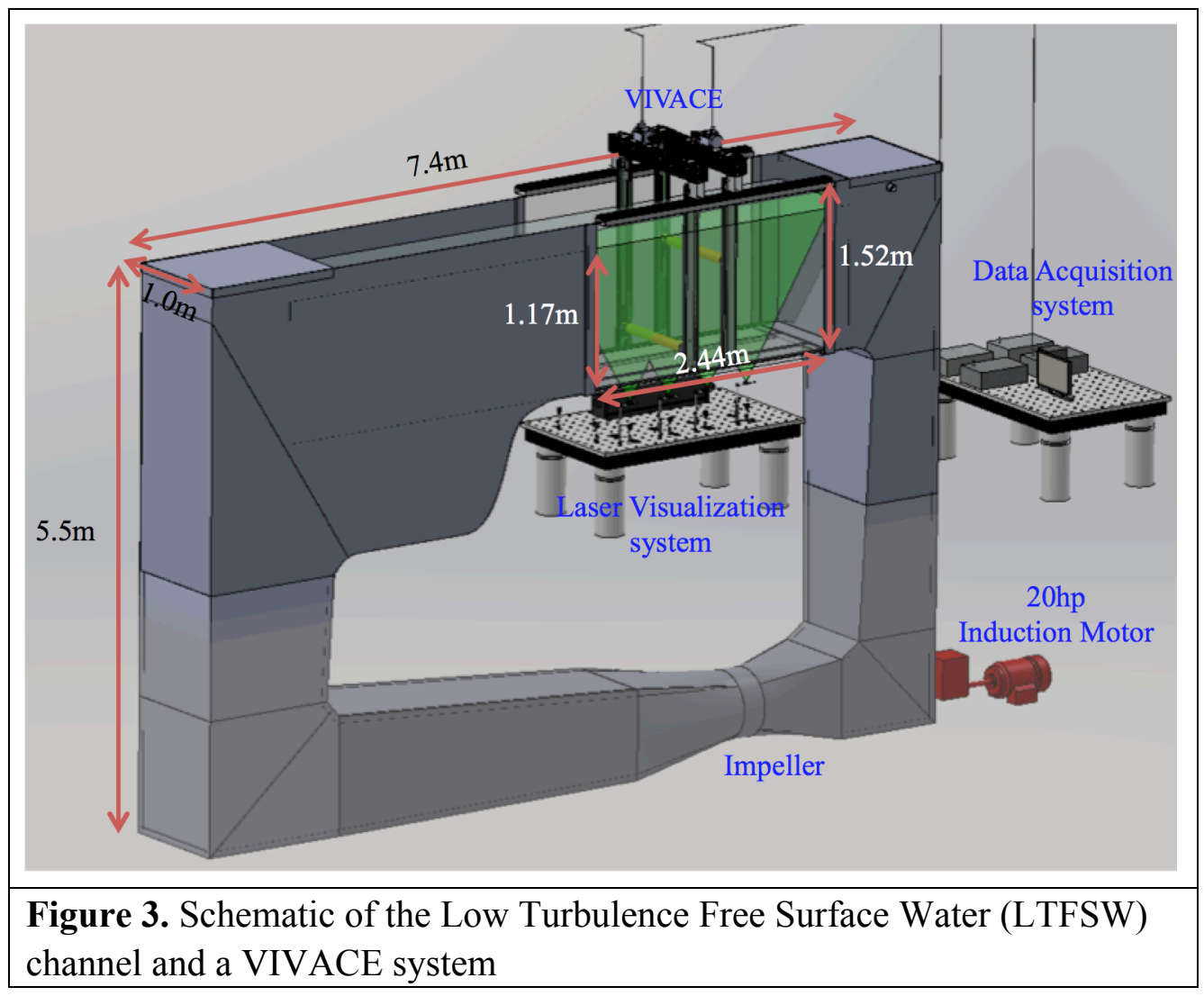

\subsection{Cylinders and distributed roughness}

A simple VIVACE Converter is a single degree-of-freedom, mass-damping-spring oscillator consisting of a single circular cylinder suspended on end-springs. Mass accounts for that of the cylinder, springs, transmission belts and pulleys, and all oscillating parts including the virtual oscillator (Vck) system (Section 2.3). Damping has two components: the mechanical transmission damping inherent in the oscillator that dissipates energy, and the external damping added for harnessing energy. It also has a power take-off (PTO) system. The excitation, comes from the fluid-structure interaction (FSI) and is applied to the cylinder through the forces imparted by the relative motion of the fluid with respect to the cylinder in FIM. 
In order to enhance the FIM of the cylinder, which results in harnessing more horizontal hydrokinetic energy, distributed roughness in the form of roughness strips was introduced and extensively studied in the MRELab experimentally (Bernitsas and Raghavan 2011, Chang et al. 2010, Park et al. 2013a, Kim et al. 2013) and numerically (Ding et al. 2013, 2015). The distributed roughness was termed Passive Turbulence Control (PTC) and this study resulted in a most valuable tool, the PTC-to-FIM Map (Park et al. 2013a, 2013b). As shown in Fig. 4, PTC consists of a pair of straight roughness strips attached symmetrically to the surface of the cylinder. According to the PTC-to-FIM Map, the location of the roughness strips determines the response FIM of the cylinder. The placement angle $\alpha_{P T C}=20^{\circ}$, is measured in degrees with respect to the forward stagnation point to the upstream edge of the roughness strip. Based on the results of previous studies (Chang et al. 2011, Park et al. 2013a) on PTC, the strips with commercial roughness designation P60 and width of $12.7 \mathrm{~mm}$, which covers 16 degrees on each side of the $88.9 \mathrm{~mm}$ diameter cylinder have been used in this study. Table 1 shows the details of the roughness strips used in the present study.

Table 1. PTC parameters (Sandpaper P60)

\begin{tabular}{|l|l|l|}
\hline Strip placement angle & $\alpha_{P T C}[$ degree $]$ & 20 \\
\hline Angular coverage of strip & $\theta[$ degree] & 16 \\
\hline Sand paper plus tape thickness & $P[\mathrm{~mm}]$ & 0.587 \\
\hline Average grit height & $k[\mathrm{~mm}]$ & 0.26 \\
\hline Total thickness of strip & $T=P+k[\mathrm{~mm}]$ & 0.847 \\
\hline
\end{tabular}




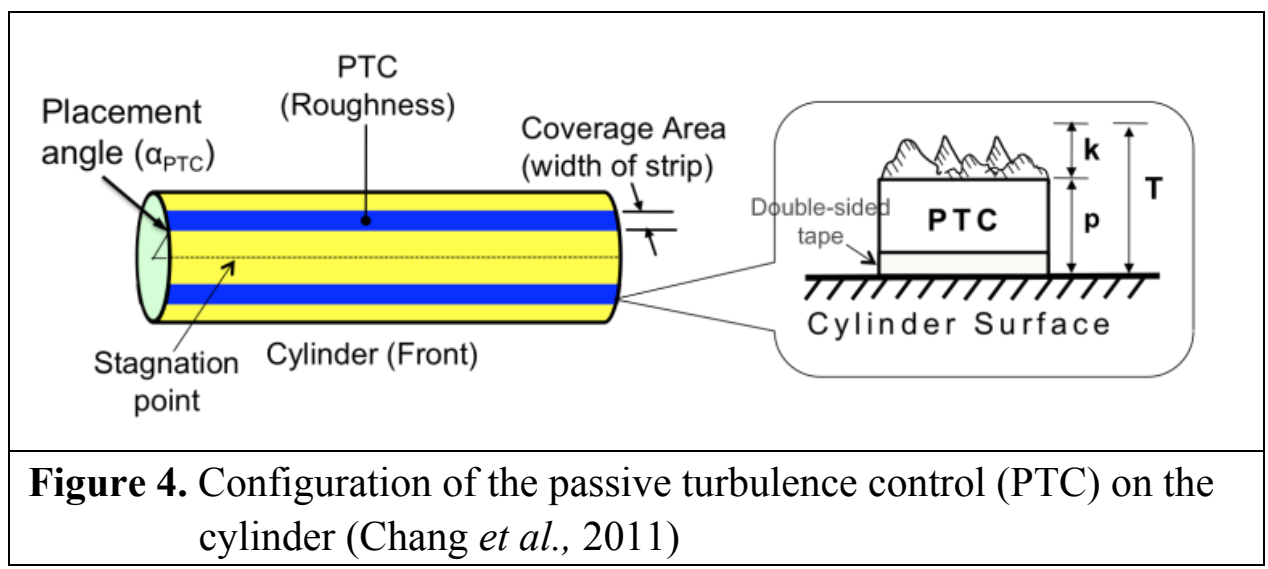

The total thickness of the PTC is on the order of the boundary layer thickness. The robustness of the PTC-to-FIM Map was studied by Park et al. (2013b) defining not only the PTC coverage requirement in the direction of the flow but also the hierarchy of dominance of the zones where PTC is applied. PTC has a major impact on the energy conversion and the efficiency of the Converter.

\subsection{Vck system}

For systematic experimental testing particularly in such a large design space, two challenges are important to overcome: (a) Changing the physical springs and dampers requires extensive preparation for calibration and alignment. (b) The viscous damping of the system is rarely linear as modeled in classical vibration textbooks. To compensate for these two major challenges, Lee and Bernitsas (2010) and Lee et al. (2011) introduced and implemented the first generation of virtual spring-damping device called Vck system. Sun et al. (2015) developed the embedded, second-generation of Vck in VIVACE oscillators. The purpose of Vck is to replace the physical 
springs and dampers of the oscillator with a simulator without including the hydrodynamics in the closed control loop, which would bias the FIM phenomenon being measured.

Several improvements were achieved in the second-generation Vck compared to the first generation: (a) The second-generation Vck system was developed as a stand-alone application via an embedded controller. (b) Digital signals were used instead of analog signals. The sample time was reduced by three orders of magnitude, thus, eliminating the need to include dynamic terms with memory in the damping model to achieve no bias in FIM and energy harnessing. Both systems are capable of compensating for the system nonlinear damping. In both Vck generations, the total nonlinear expression for the viscous damping of the system is modeled and measured after extensive system identification and is subtracted from the system through the controller to nullify the system damping. Subsequently, a mathematically linear viscous damping is introduced. This is hardly achievable in engineering applications; and yet it is used in modeling oscillators in textbooks. With the Vck controller, perfectly linear viscous damping is achieved and implemented. A resistor bank is used to dissipate the generated power.

The most notable achievement of the Vck system is that it does not include the hydrodynamic force in the closed control loop, and thus, does not bias the measured phenomenon. As a result, Vck simulates the oscillator only and not the FIM phenomenon. This is achieved at the expense of extensive system identification of the real system viscous damping. System identification for a totally new system may require several months of modeling and measurements using free decay tests and error minimization. 
Test-models were redesigned and built recently as shown in Figs. 5-6. In the new design, the linear motion mechanism was brought under water, which resulted in most of the Converter components being under water. The whole linear motion system, which consists of the end sliding-blocks, one timing-belt and pulleys, is fitted inside of a $38 \mathrm{~mm}$ wide slender tube. The sliding block connects the cylinder to the Vck system through two timing belts and pulleys as shown in Fig. 6. The new system better models real, field-deployed, devices compared to previous models tested in the MRELab, where only the oscillating cylinders and supporting end-struts were under water. The structural differences result in stronger tip-flow effects in the new system (Kinaci et al., 2016).

The mass ratio $m^{*}$ is changed by placing a mass-shaft inside the cylinder (Sun et al., 2015). The center-to-center distance $(d / D)$ between cylinders in tandem in the flow direction can be adjusted by the rail system starting at $5 "(12.7 \mathrm{~mm})$ in increments of $0.5 "(1.27 \mathrm{~mm})$. The features of the new VIVACE Converter are summarized in Table 2.

Table 2. Specifications of the new VIVACE Converter

\begin{tabular}{|c|c|c|c|}
\hline Mass Ratio & Aspect Ratio & Maximum Oscillatory Amplitude & Longitudinal Distance \\
\hline $0.634 \leq m^{*} \leq 2.00$ & $L / D=10.07$ & $A_{\max } / D_{3.5^{\prime \prime}}=5.5$ & $1.429 \leq d / D_{3.5^{\prime \prime}} \leq 6.0$ \\
\hline
\end{tabular}



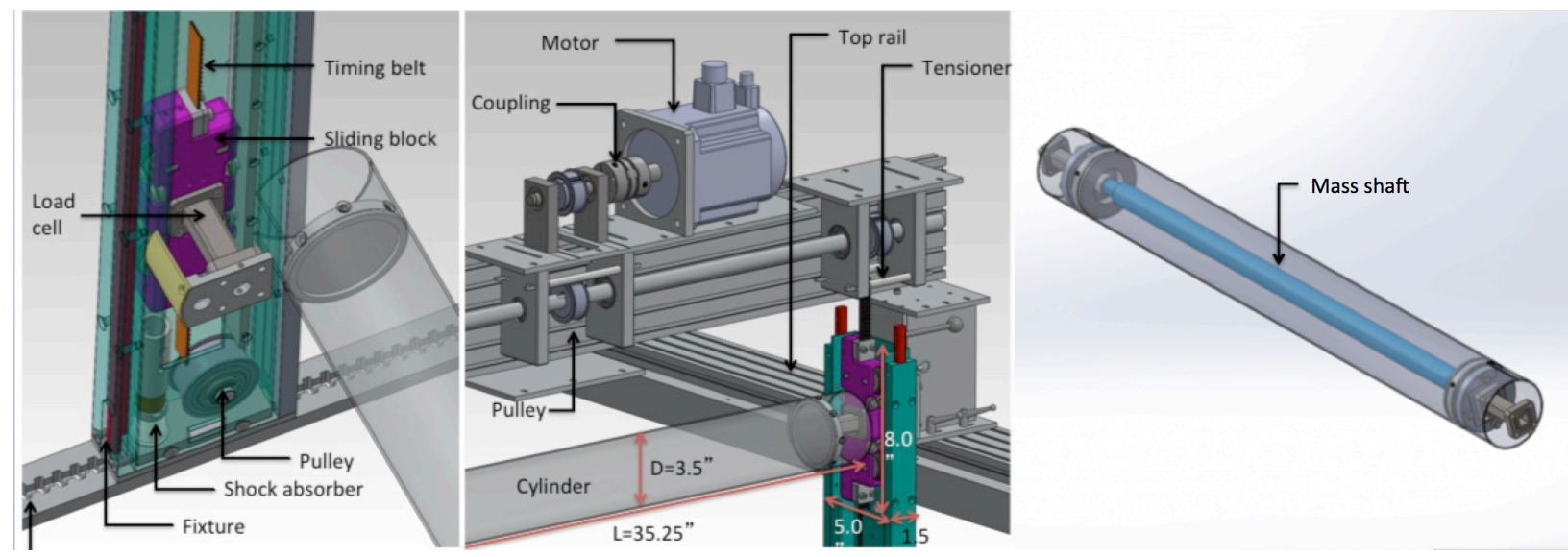

Figure 5. Details of the new VIVACE Converter

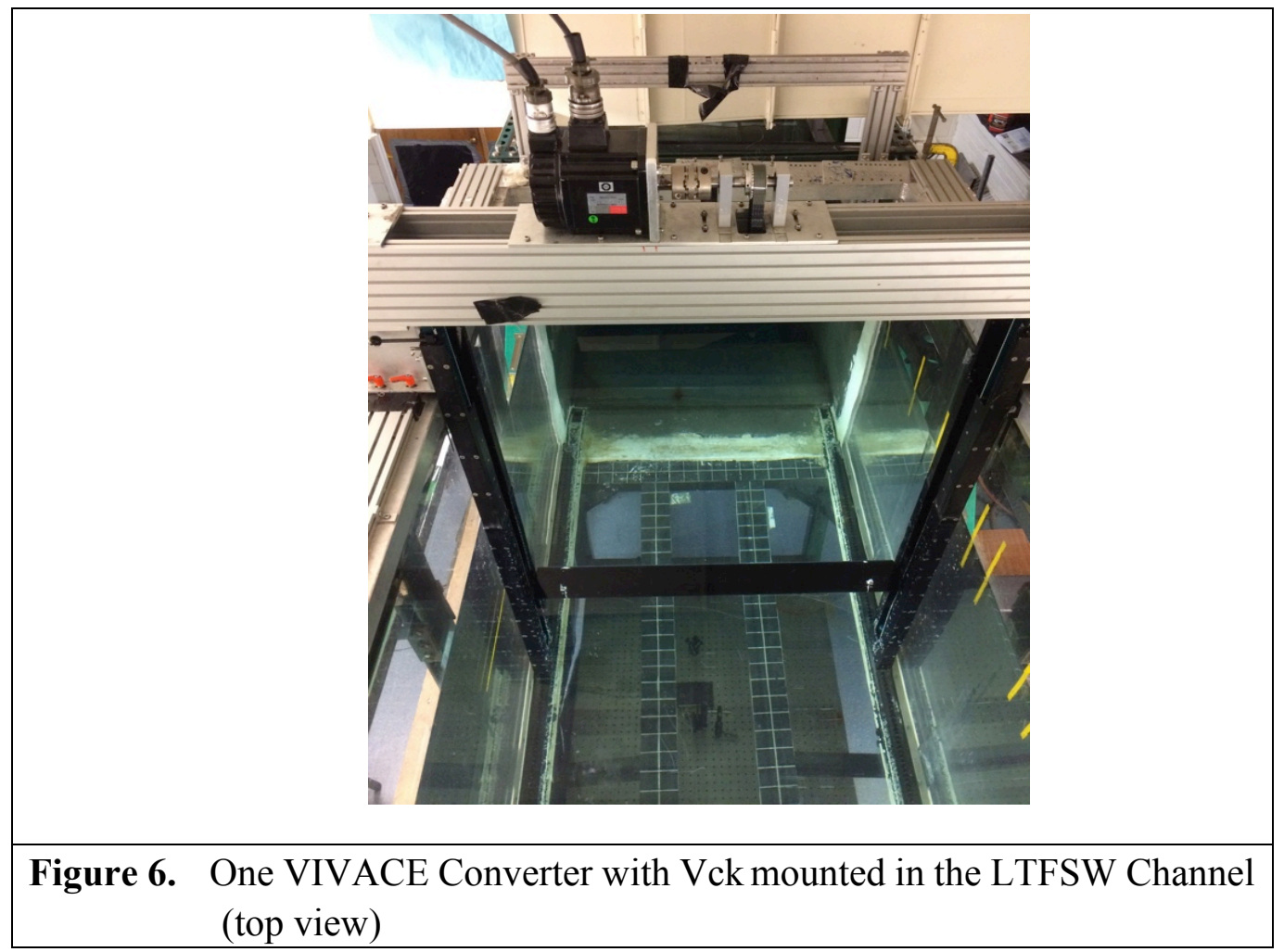

The main function of the Vck system is to add mathematically accurate damping and stiffness models. In this case, a linear, piece-wise continuous, viscous adjustable damping model 
is developed and added to the system to harness hydrokinetic energy. The end product is a power envelope for which systematic tests must be conducted. Prior to adding prescribed damping, the physical system damping needs to be identified and subtracted. The latter is due to friction in all moving parts, especially in the timing belts and pulleys, which bring undesirable/excessive amount of nonlinear damping to Vck. That results in dissipated (lost) energy to overcome the system damping (friction).

Based on the non-linear viscous damping model, the controller force can be expressed as:

$$
F_{\text {controller }}=F_{\text {nonlinear_damping }}-c_{\text {vitual }} \dot{y}-K_{\text {virtual }} y
$$

where $F_{\text {controller }}$ is the force applied by the virtual system (motor and controller), $F_{\text {nonlinear_damping }}$ is the total nonlinear damping force, $K_{\text {virtual }}$ is the virtual, spring constant or prescribed function, $c_{\text {virtual }}$ is the virtual linear viscous damping. Regarding damping, a prescribed $c_{\text {virtual }}$ function is added after subtracting the nonlinear viscous damping of the system, so that the system tested has a damping model mathematically-true as prescribed. The agreement between the real spring system and Vck system is very good in VIV and galloping measurements over the entire range of velocities tested, has been well documented by Sun et al. (2015).

\subsection{Nonlinear Restoring Force simulated by Vck}

The objective of VIVACE is to convert hydrokinetic energy to mechanical power in the oscillator and then to electrical power through the generator. The latter is essentially energy dissipated through additional damping. In the VIV region, the cylinder needs soft spring in order 
to initiate VIV earlier. Higher spring stiffness, however, results in higher natural frequency and higher power harnessed in the upper branch (Bernitsas et al. 2012). Galloping, on the other hand, is an instability phenomenon, where, as the stiffness decreases, the oscillation amplitude increases resulting in higher energy harnessing (Sun, et.al. 2015, Chang, et.al. 2013). The response of lower stiffness need to be constrained for safety reasons and in order to achieve optimal power harness. The key aspect of nonlinear springs is to set a piecewise spring that can initiate VIV in relatively lower flow velocity and high amplitude response in galloping, and can also have higher spring constant at the ends of oscillation, acting as energy absorbing safety bumpers. The higher spring stiffness in the end-bumpers is essentially an efficient mechanism to reverse the linear momentum of the oscillating cylinder at the end of the travel determined by the frame of the oscillator and the test section of the facility. So the following piecewise function of nonlinear spring/restoring force are simulate through Vck system:

$$
\text { Nonlinear restoring force }=\left\{\begin{array}{l}
K_{1} \times y, \text { if }|y| \leq y_{0} \\
\left(K_{1} \times y\right) \times y_{0}+K_{2} \times\left(y-y_{0}\right), \text { if }|y| \quad y_{0}
\end{array}\right.
$$

where, $K_{1}$ and $K_{2}$ are set to be $200 \mathrm{~N} / \mathrm{m}$ and $1,000 \mathrm{~N} / \mathrm{m}$, respectively; $y_{0}$ is the transition position where the spring stiffness value changes between $K_{1}$ and $K_{2}$ as shown in Figure 8. 


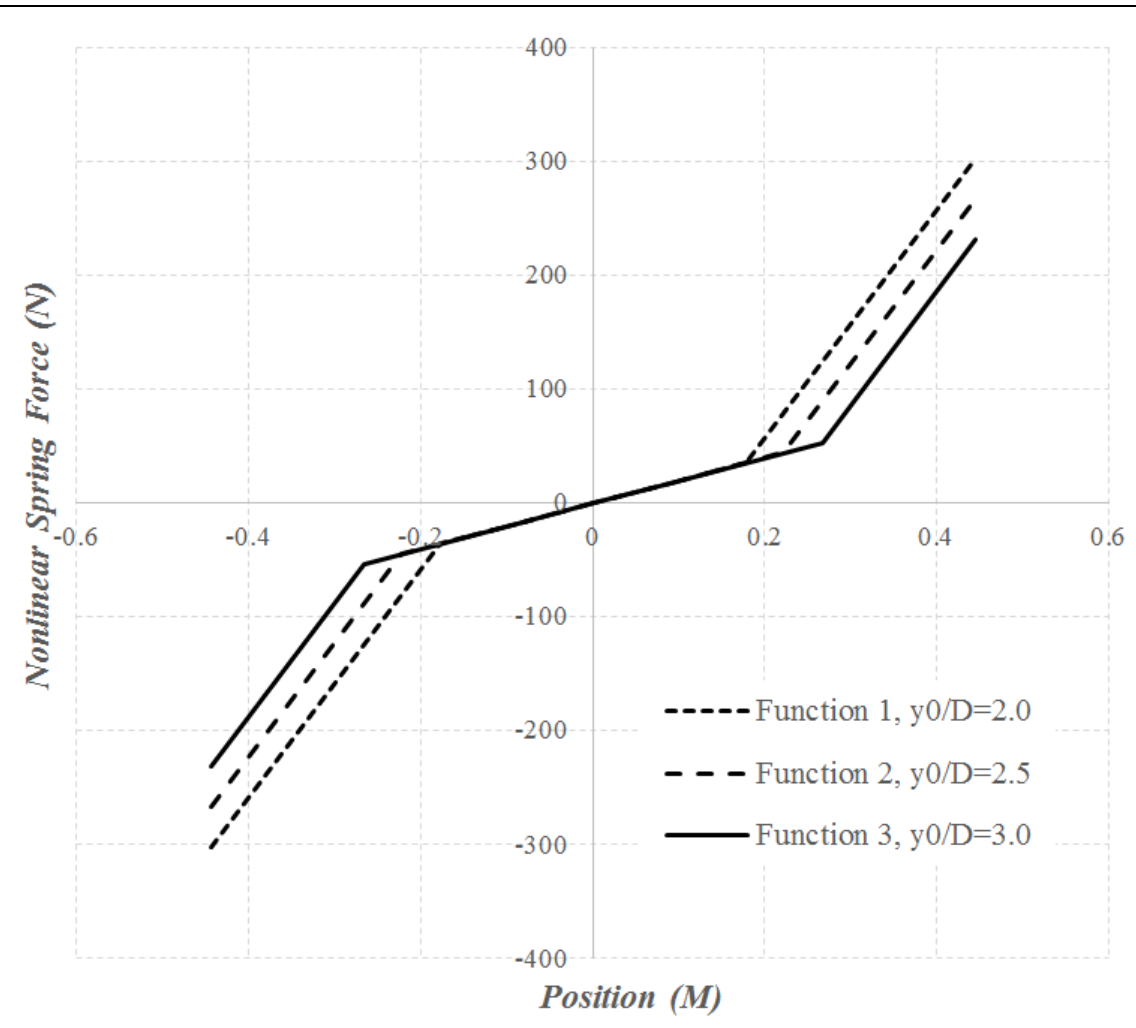

Figure 7. Nonlinear piecewise stiffness functions

The main objective of nonlinear-spring restoring-force is to establish the "soft" initial stage, and the "bumpers" at two ends. The latter are beneficial in galloping where the main driving mechanism is insatiability. If the frame of the oscillator were infinitely wide, the oscillating cylinder would have infinite travel space, the PTC cylinder would be driven by the shear-layer alternating instability, and the cylinder speed would increase until a steady state is reached. The purpose of establishing the nonlinear function in equation (5), is twofold: First, allow VIV onset at lower flow speed. Second, enable adjustment of the $y_{0}$ position (swapping between $K_{l}$ and $K_{2}$ ), to find the optimal $y_{0}$ to allow maximum oscillator speed with no loss of energy at the end of the travel due to collision at the end of the travel. 


\section{MATHEMATICAL MODEL OF HARNESSED AND DISSIPATED POWER}

Assuming that the flow velocity in is the $x$-direction, the motion of the cylinder is in the $y$-direction (vertical), the cylinder axis is horizontal in the $z$-direction, and the oscillator dynamics can be modeled by a second order linear differential equation, we have

$$
m_{\text {osc }} \ddot{y}+c_{\text {total }} \dot{y}+K y=F_{\text {fluid }} y
$$

where $y$ is the direction perpendicular to the flow and the cylinder axis, $m_{o s c}$ is the total oscillating system mass, which includes one third of the spring mass, $K$ is the spring stiffness, $c_{\text {total }}$ is the total damping coefficient, and $F_{\text {fluid }}$ is the force exerted by the fluid on the cylinder in the $y$-direction.

In order to convert hydrokinetic energy to mechanical energy in the oscillator and subsequently electrical energy, additional damping is introduced into the system. The total damping $c_{\text {total }}$ is defined as

$$
c_{\text {total }}=c_{\text {structure }}+c_{\text {harness }}
$$

where $c_{\text {structure }}$ is the existing damping due to losses in the transmission system, $c_{\text {harness }}$ is the damping added through Vck that converts the mechanical energy in the oscillating cylinder to electrical energy simulating the real world. In equation (7), $c_{\text {structure }}$ and $c_{\text {harness }}$ can be expressed using the damping ratio $\zeta_{\text {harness }}$ and $\zeta_{\text {structure }}$, respectively, as

$$
\zeta_{\text {structure }}=\frac{c_{\text {structure }}}{2 \sqrt{\left(m_{\text {osc }}+m_{a}\right) K}}
$$




$$
\zeta_{\text {harness }}=\frac{c_{\text {harness }}}{2 \sqrt{\left(m_{\text {osc }}+m_{a}\right) K}}
$$

Then, the mechanical power in VIVACE can be expressed as:

$$
P_{\text {converted }}=\frac{1}{T_{\text {osc }}} \int_{0}^{T_{\text {osc }}} 4 \pi\left(m_{\text {osc }}+m_{a}\right) \zeta \dot{y}^{2} f_{n, \text { water }} d t=8 \pi^{3}\left(m_{\text {osc }}+m_{a}\right) \zeta_{\text {total }}\left(A f_{\text {osc }}\right)^{2} f_{n, \text { water }}
$$

Using equation (10), we obtain the harnessed power and the dissipated power as

$$
\begin{aligned}
& P_{\text {dissipated }}=8 \pi^{3}\left(m_{\text {osc }}+m_{a}\right) \zeta_{\text {structure }}\left(A f_{\text {osc }}\right)^{2} f_{n, \text { water }} \\
& P_{\text {harness }}=8 \pi^{3}\left(m_{\text {osc }}+m_{a}\right) \zeta_{\text {harness }}\left(A f_{\text {osc }}\right)^{2} f_{n, \text { water }}
\end{aligned}
$$

Since equations (11) and (12) appear to depend explicitly on the added mass $m_{a}$, it is worth noting that they can be recast as follows, respectively.

$$
P_{\text {dissipated }}=\frac{1}{2} c_{\text {structure }} A^{2} \omega_{\text {osc }}^{2}
$$

and

$$
P_{\text {harness }}=\frac{1}{2} c_{\text {harness }} A^{2} \omega_{\text {osc }}^{2}
$$

where, $A$ is the amplitude of the cylinder, $\omega_{o s c}$ is the angular frequency of oscillation, which can be measured using the Vck system. These two equations do not depend on the specific way the added mass is calculated or even the value of the added mass explicitly. For detailed derivation of the above equations, the reader is referred to (Sun, et al. 2016). 


\section{RESULTS AND DISSCUSSION}

A series of experiments are conducted under three different nonlinear spring stiffness models, and harnessing linear-viscous damping of $10 \mathrm{Ns} / \mathrm{m} \leq c_{\text {harness }} \leq 40 \mathrm{Ns} / \mathrm{m}$. It should be noted that the mathematical model of the nonlinear spring stiffness is accurate in form and value because it is defined by the calibrated motor-torque and its relation to rotation. That is, the spring value is not subjected to fabrication error or degradation in time due to fatigue. Similarly, the values of damping are exact and the corresponding linear viscous function is accurate as explained in the discussion on the Vck system. The Reynolds number range is $24,000 \leq R e \leq 120,000$. The particulars for each case are shown in Table 4.

Table 4 Particulars of single-cylinder VIVACE model

\begin{tabular}{|l|l|l|l|}
\hline & Test set 1 & Test set 2 & Test set 3 \\
\hline$K_{1}[\mathrm{~N} / \mathrm{m}]$ & 200 & 200 & 200 \\
\hline$K_{2}[\mathrm{~N} / \mathrm{m}]$ & 1,000 & 1,000 & 1,000 \\
\hline$y_{0} / D$ & 2.0 & 2.5 & 3.0 \\
\hline$c_{\text {harness }}[\mathrm{Ns} / \mathrm{m}]$ & $10,20,30,40$ & $10,20,30,40$ & $10,20,30,40$ \\
$\zeta_{\text {harness }}=\frac{c_{\text {harness }}}{2 \sqrt{K_{1} m_{\text {osc }}}}$ & $0.13,0.26$, & $0.13,0.26$, & $0.13,0.26$, \\
& $0.39,0.53$ & $0.39,0.53$ & $0.39,0.53$ \\
\hline$c_{\text {structure }}[N s / \mathrm{m}]$ & 1.53 & 1.53 & 1.53 \\
\hline$\zeta_{\text {structure }}=\frac{c_{\text {structure }}}{2 \sqrt{K_{1} m_{\text {osc }}}}$ & 0.02 & 0.02 & 0.02 \\
\hline$D[\mathrm{~m}]$ & 0.0889 & 0.0889 & 0.0889 \\
\hline$L[\mathrm{~m}]$ & 0.895 & 0.895 & 0.895 \\
\hline$m_{\text {dis }}=\rho \pi D^{2} / 4[\mathrm{~kg}]$ & 5.425 & 5.425 & 5.425 \\
\hline$m_{\text {osc }}[\mathrm{kg}]$ & 7.286 & 7.286 & 7.286 \\
\hline$m_{a}[\mathrm{~kg}]$ (inviscid) & 5.425 & 5.425 & 5.425 \\
\hline Temperature $\left[{ }^{\circ} \mathrm{C}\right]$ & 20.5 & 20.5 & 20.5 \\
\hline
\end{tabular}




\begin{tabular}{|l|l|l|l|}
\hline$\mu\left[N \mathrm{~s} / \mathrm{m}^{2}\right]$ & $1.004 \mathrm{E}-03$ & $1.004 \mathrm{E}-03$ & $1.004 \mathrm{E}-03$ \\
\hline$v\left[\mathrm{~m}^{2} / \mathrm{s}\right]$ & $9.940 \mathrm{E}-07$ & $9.940 \mathrm{E}-07$ & $9.940 \mathrm{E}-07$ \\
\hline$\rho\left[\mathrm{kg} / \mathrm{m}^{3}\right]$ & 999.729 & 999.729 & 999.729 \\
\hline
\end{tabular}

\subsection{Oscillatory Response}

The amplitude ratio $A / D$ and the frequency response are plotted versus Reynolds number $R e$ and flow velocity $U$ in Figs. 8-10 for the PTC-cylinder. The amplitude $A$ of the cylinder is calculated by averaging the absolute values of the 30 highest values of positive and 30 highest negative peaks in 60 seconds of running time past the transient. The mean absolute deviation is denoted by vertical error bars.

The oscillation frequency $f_{\text {osc }}$ is calculated by FFT (Fast Fourier Transform) of the displacement of the cylinder over the recorded period with the exclusion of the end-transients. The frequency is not affected significantly by the total damping ratio in the VIV region. On the contrary, there is significant variation of the frequency of oscillation in the transition from VIV to galloping and in fully developed galloping due to variation of the linear-viscous damping

Due to the low stiffness of the first stage of the nonlinear restoring force $\left(K_{l}=200 \mathrm{~N} / \mathrm{m}\right.$, $K_{2}=1,000 \mathrm{~N} / \mathrm{m}$ ), a distinctive gap between VIV and galloping can be observed. In previous experiments with linear spring stiffness (Sun et al. 2015, Park et al. 2013a), it was shown that the gap can be closed by either increasing the initial linear stiffness, thus delaying VIV onset, or reducing damping thus initiating galloping earlier. This can be verified using equations (1)-(3). 
The gap can also be bridged by changing the PTC location, but this is beyond the scope of this research (Park et al. 2013b).

In all three cases (Figs. 8-10), it can be observed that the amplitude response in galloping increases considerably compared to the linear-spring amplitude. Four regions can be observed in the amplitude ratio curves. It should be noted that, the VIV amplitude response of the nonlinear spring tests is within the range of the $K_{l}$ linear spring segment $\left(y_{0} / D=2.0,2.5,3.0\right)$. 

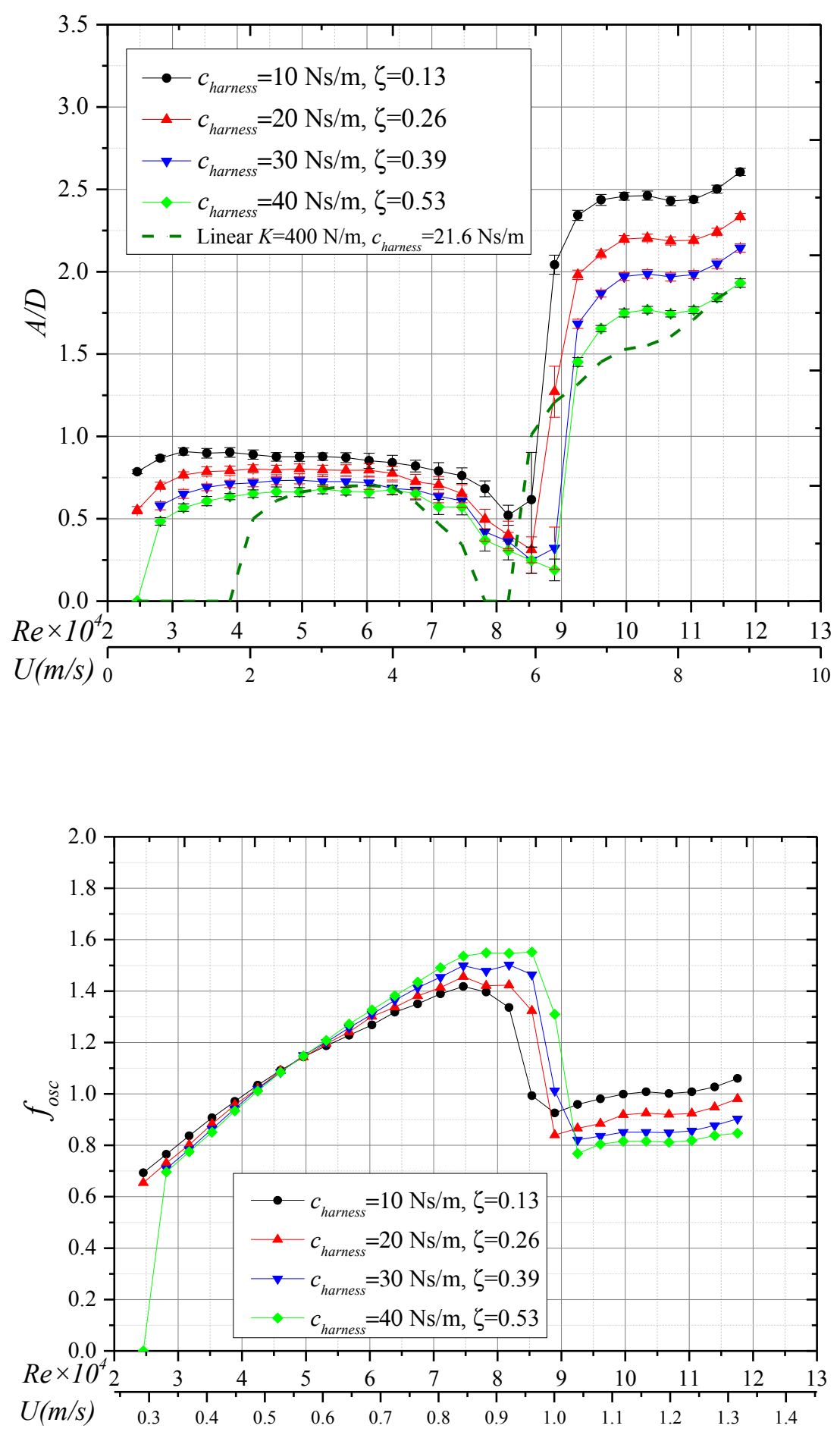

Figure 8. PTC-cylinder oscillatory response for nonlinear restoring force $K_{l}=200 \mathrm{~N} / \mathrm{m}$ and $K_{2}=1,000 \mathrm{~N} / \mathrm{m}$; four harness damping values $\mathrm{c}_{\text {harness }}$; the corresponding damping ratio to $K_{1,} \zeta$ $=0.13,0.26,0.39,0.53$; mass ratio $m^{*}=1.343$; nonlinear piecewise function $y_{0} / D=2.0$. 

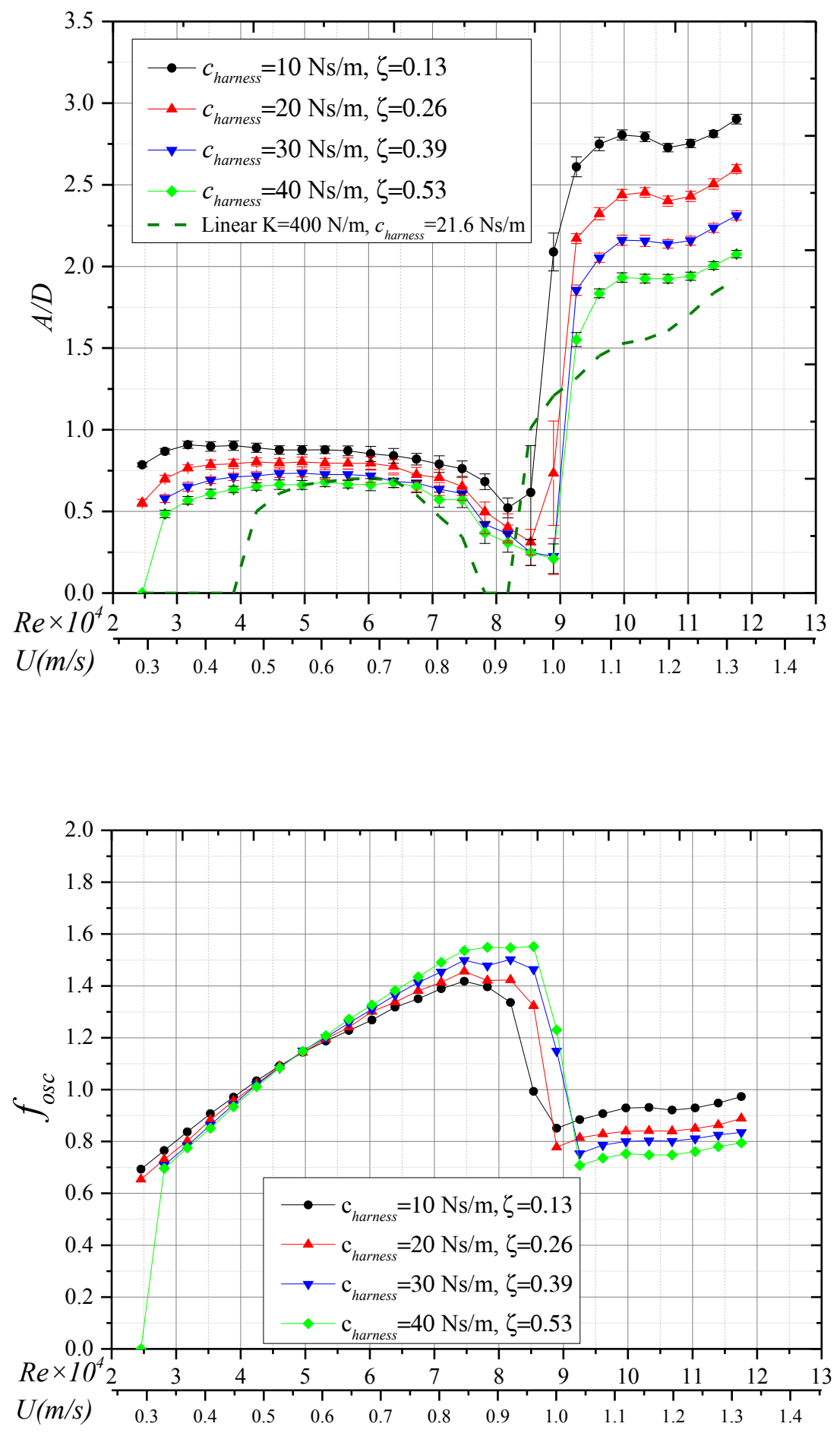

Figure 9. PTC-cylinder oscillatory response for nonlinear restoring force $K_{l}=200 \mathrm{~N} / \mathrm{m}$ and $K_{2}=1,000 \mathrm{~N} / \mathrm{m}$; four harness damping values $\mathrm{c}_{\text {harness }}$; the corresponding damping ratio to $K_{1}, \zeta$ $=0.13,0.26,0.39,0.53$; mass ratio $m^{*}=1.343$; nonlinear piecewise function $y_{0} / D=2.5$. 

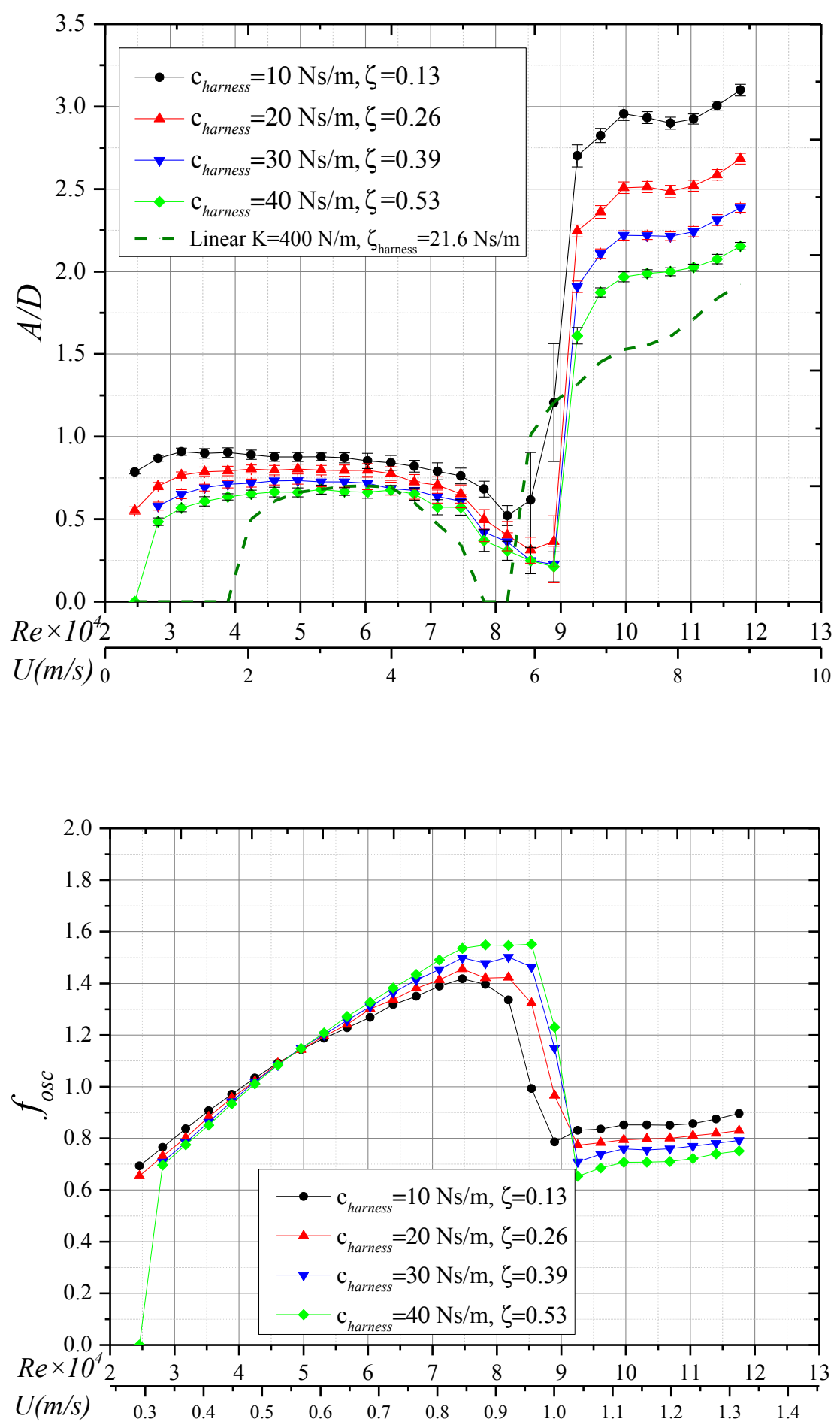

Figure 10. PTC-cylinder oscillatory response for nonlinear restoring force $K_{l}=200 \mathrm{~N} / \mathrm{m}$ and $K_{2}=1,000 \mathrm{~N} / \mathrm{m}$; four harness damping values $\mathrm{c}_{\text {harness }}$; the corresponding damping ratio to $K_{1}, \zeta$ $=0.13,0.26,0.39,0.53$; mass ratio $m^{*}=1.343$; nonlinear piecewise function $y_{0} / D=3.0$. 
(a) $25,000 \leq R e \leq 30,000$ : Is the initial branch of VIV for all the stiffness. A drop in $A / D$ can also be observed for all stiffness values as $c_{\text {harness }}$ increases from $10 N s / m$ to $40 \mathrm{Ns} / \mathrm{m}$ corresponding to really large values of $\zeta$ from 0.13 to 0.53 . This is due to additional energy harvested. The frequency increases linearly in this region except for $c_{\text {harness }}=40 \mathrm{Ns} / \mathrm{m}$, where the high damping delays the onset of VIV. At $R e=30,000$, the $c_{\text {harness }}=40 \mathrm{Ns} / \mathrm{m}$ has slightly lower frequency compared to $c_{\text {harness }}=10 \mathrm{Ns} / \mathrm{m}$.

(b) $30,000 \leq R e \leq 70,000$ : This is the upper branch of VIV for all the spring stiffness functions tested. It should be pointed out, that for all the nonlinear functions the upper branch starts at the same $R e=30,000$ due to the low linear spring. The amplitude in the upper branch stays flat and shows a downward trend. The error bars are shorter at the beginning of the upper branch indicating that VIV is more stable compared to the end of the upper branch. The frequency response shows a sharply upward trend $\left(f_{\text {osc }}=0.65 \mathrm{~Hz}\right.$ to $f_{\text {osc }}=1.55$ at $\left.c_{\text {harness }}=40 \mathrm{Ns} / \mathrm{m}\right)$. It should also be noticed that at $R e=50,000$, the frequency response of higher harness damping increases drastically and exceeds the frequency response at lower harnessing damping, which is beneficial for power harnessing as evident in equation (14).

(c) $70,000 \leq \operatorname{Re} \leq 85,000 \sim 87,000$ : This range includes the lower branch of VIV, the desynchronization range, the gap between VIV and galloping, and the onset of galloping. All four sub-ranges are obvious in the amplitude and frequency responses in Figs. 8-10. Increasing of damping $c_{\text {harness }}$ shrinks the VIV range and delays the onset of galloping separating VIV from 
galloping. In this region which includes all four sub-ranges, the amplitude drops dramatically and random oscillatory patterns appear as expected. Consequently, the error bars become significantly larger in this region as shown in Figs. 8-10.

As Re approaches 80,000, the frequency drop in Figs. 8-10 indicates transition to galloping. In the frequency plots, no gap occurs between the upper branch in VIV and galloping. The frequency drop is accompanied by a rapid increase in the amplitude of the cylinder. In this region, the frequency response increases with increasing damping. Thus, high damping $c_{\text {harness }}=40 \mathrm{Ns} / \mathrm{m}$ results in $f_{\text {osc }}=1.55 \mathrm{~Hz}$ at $R e=85,000$ compared to $f_{\text {osc }}=1.41 \mathrm{~Hz}$ at $R e=75,000$, $c_{\text {harness }}=10 \mathrm{Ns} / \mathrm{m}$; both are at their corresponding end of the upper VIV branch.

(d) $87,000-94,000 \leq R e$ : This is the galloping region. Galloping is known to be a high-amplitude and low-frequency FIM. The galloping generation mechanism is based on shear layer instability due to asymmetry. Non-circular cross-section cylinders may be subjected to high amplitude galloping oscillation due to their geometric asymmetry with respect to the flow. In this study, the roughness strips result in geometric asymmetry, thus, inducing galloping.

The following observations can be made in this region: (i) The amplitude increases dramatically with the onset of galloping. (ii) The $K_{2}$ stiffness also affects the speed of oscillation of the cylinder, which is proportional to $A \omega_{\text {osc }}$. Both $A$ and $\omega_{\text {osc }}$ increase in the galloping region as shown in Figs. 8-10. For example, when restoring Function 1 (Fig. 7) is used, the frequency of oscillation $f_{\text {osc }}$ changes from $0.9 \mathrm{~Hz}$ to $1.1 \mathrm{~Hz}$ and $A$ increases from 2.15 to 2.75 at $c_{\text {harness }}=10 \mathrm{Ns} / \mathrm{m}$. Similarly for $c_{\text {harness }}=40 \mathrm{Ns} / \mathrm{m} f_{\text {osc }}$ changes from $0.76 \mathrm{~Hz}$ to $0.87 \mathrm{~Hz}$ and $A$ 
increases from 1.55 to 2.10. (iii) All three restoring force functions used are effective as the amplitude of oscillation reaches the $K_{2}$ segment. Specifically, $A / D=2.61$ for Function 1 with $y_{0} / D=2.0 ; A / D=2.90$ for Function 2 with $y_{0} / D=2.5$; and $A / D=3.1$ for Function 3 with $y_{0} / D=3.0$.

\subsection{Frequency Content of Oscillations}

In order to further investigate the FIM nature of piecewise nonlinear stiffness, the FFT of the time history of the response amplitude for restoring Function $1\left(y_{0} / D=2.0\right)$ is presented in Fig. 11 for $c_{\text {harness }}=10 \mathrm{Ns} / \mathrm{m}$ and $c_{\text {harness }}=40 \mathrm{Ns} / \mathrm{m}$ as function of the flow velocity. The $y$-axis is the flow velocity $U$. The red dash line is the Strouhal frequency; the blue dash line connects the peak frequency at each velocity; and the black line corresponds to 1 for comparison.

In the VIV initial branch, the oscillation frequency follows the Strouhal frequency. Resonance starts at the upper VIV branch as a typical PTC-cylinder response (Eun Soo Kim, et.al. 2013). This is due to the fact that the $K_{2}$ segment of the nonlinear restoring force has not been reached making the oscillator a linear oscillator in the $K_{l}$ segment. There is a single dominant frequency shown in Fig. 11 for both harnessing damping values. In the lower VIV branch and in desynchronization, at $0.9 \mathrm{~m} / \mathrm{s}<U<1.0 \mathrm{~m} / \mathrm{s}$, multiple frequencies can be observed at lower harnessing damping ( $c_{\text {harness }}=10 \mathrm{Ns} / \mathrm{m}$ ), but not at higher harnessing damping $\left(c_{\text {harness }}=40 \mathrm{Ns} / \mathrm{m}\right)$, where oscillation ceases.

The frequency content of FFT in galloping appears different to typical PTC-cylinder response, where the $f_{\text {osc }}$ is nearly constant (Hai Sun, et.al. 2015). With the nonlinear restoring 
force, $f_{o s c}$ steadily increases as the flow velocity increases. In galloping, the amplitude outgrows the $K_{1}$ segment of the restoring force, it reaches the $K_{2}$ segment, and the cylinder bounces off the stiff springs $K_{2}$. Thus, in the limited space allowed by the oscillator frame, better use of galloping is achieved, which does not depend on the spring stiffness and can convert more power that VIV.

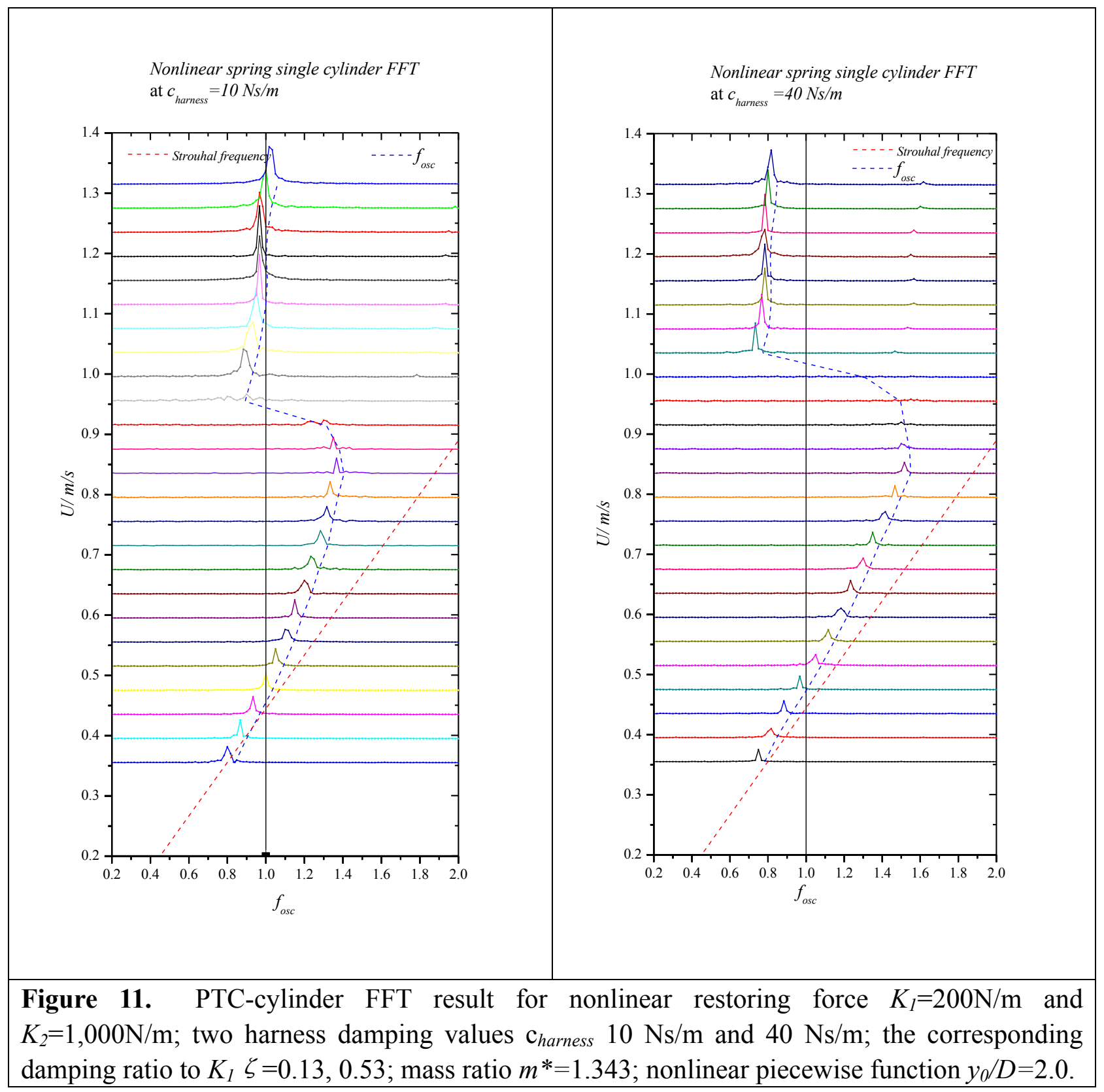


One noticeable feature is that regardless of whether the oscillator FIM is in the galloping or the VIV region, there is only one predominant frequency. There is a second, albeit small peak, around $1.4 \sim 1.7 \mathrm{~Hz}$ for $c_{\text {harness }}=40 \mathrm{Ns} / \mathrm{m}$, and $1.6 \sim 2.0 \mathrm{~Hz}$ for $c_{\text {harness }}=10 \mathrm{Ns} / \mathrm{m}$. This frequency is close to the natural frequency in water corresponding to the $K_{2}$ segment of the nonlinear restoring force. These results indicate that the oscillation is sinusoidal with a single predominant frequency, and thus, equations (11) to (14) can be used to calculate the harnessed and converted power.

\subsection{Envelope of Harnessed and Converted Power}

Experimental results for the harnessed power and converted power, for each of the three nonlinear spring-stiffness functions, and four harnessing damping values, for a PTC-cylinder are plotted versus Reynolds number and flow velocity in Fig. 12. The right $y$-axis is the scale for the total converted power, which includes both the harnessed power and the dissipated power. The latter is due to the structure/friction damping. The red dashed-line and the black dashed-line are

the power harnessed by linear spring $K=400 \mathrm{~N} / \mathrm{m}$ at $c_{\text {harness }}=21.6 \mathrm{Ns} / \mathrm{m}\left(\zeta_{\text {harness }}=0.20\right)$ and $K=1,000 \mathrm{~N} / \mathrm{m}$ at $c_{\text {harness }}=34.2 \mathrm{Ns} / \mathrm{m}\left(\zeta_{\text {harness }}=0.20\right)$, respectively, for comparison.

In Fig. 12, the following established FIM regions are easily identifiable. 


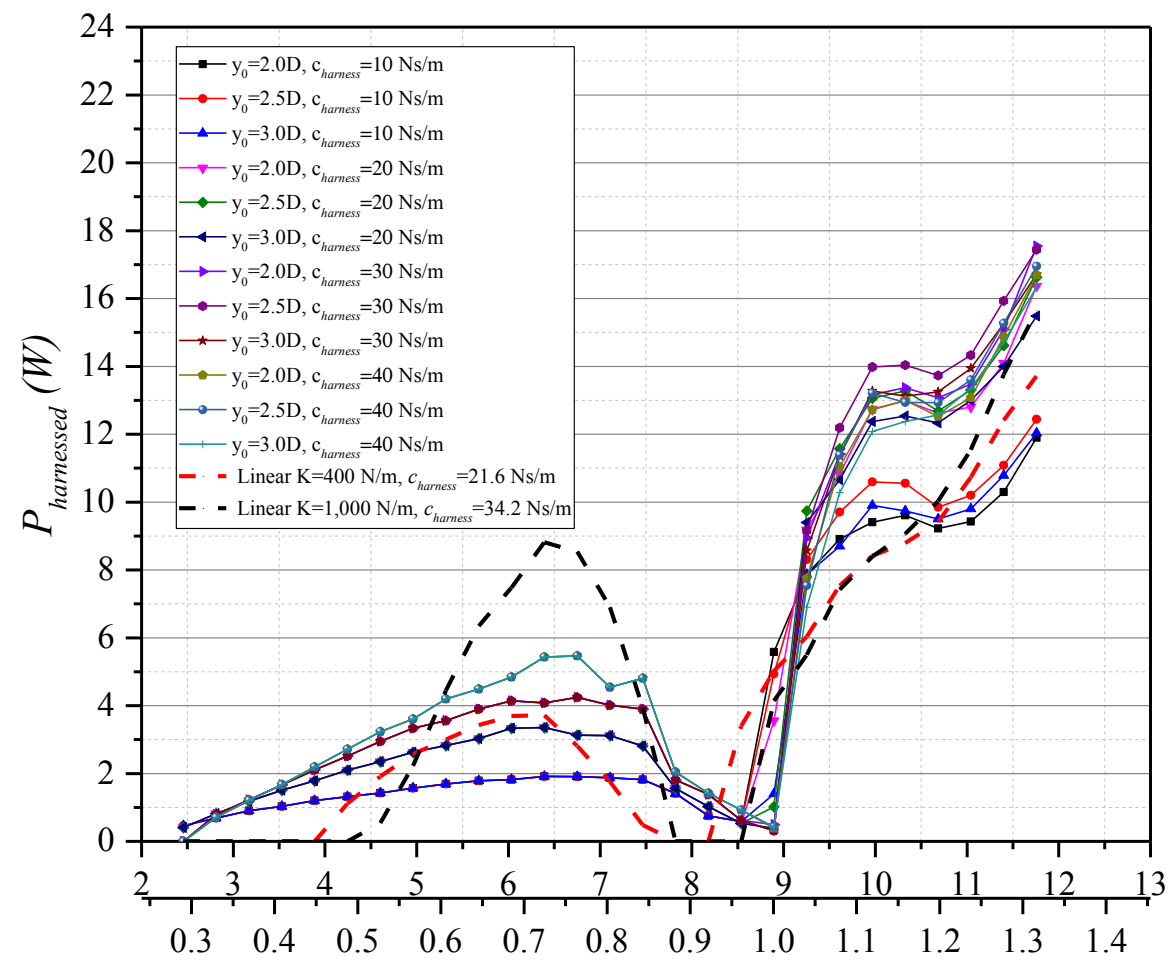

Figure 12. Harnessed and converted power for nonlinear restoring force $K_{l}=200 \mathrm{~N} / \mathrm{m}$ and $K_{2}=1,000 \mathrm{~N} / \mathrm{m} ; 4$ harness damping values $\mathrm{c}_{\text {harness }}$; the corresponding damping ratio to $K_{1}$ are $\zeta=0.13,0.26,0.39,0.53$; mass ratio $m^{*}=1.343$; nonlinear piecewise function $y_{0} / D=2.0,2.5$ and 3.0 .

(a) 23,000<Re $<68,000 \sim 70,000$ : This region contains the VIV initial branch and VIV upper branch. A steady growth of power can be observed. Initiation of power harnessing starts at low flow velocity of $0.275 \mathrm{~m} / \mathrm{s}$ due to the softer first stage of the nonlinear stiffness. The difference between different damping values is very small expected at the lowest speed.

At the upper VIV branch, the harnessed power increases as the harnessing damping increases. A trend of reduction in the increase of power can be observed between $\mathrm{c}_{\text {harness }}$ values 
indicating that a local optimum is being approached for a given flow velocity. The highest power harnessed at the VIV upper branch for $\mathrm{c}_{\text {harness }}=40 \mathrm{Ns} / \mathrm{m}, 30 \mathrm{Ns} / \mathrm{m}, 20 \mathrm{Ns} / \mathrm{m}, 10 \mathrm{Ns} / \mathrm{m}$ are $5.46 \mathrm{~W}$, $4.24 \mathrm{~W}, 3.36 \mathrm{~W}, 1.97 \mathrm{~W}$, respectively. Compared to the two linear spring results, the nonlinear stiffness initiates power harnessing at lower flow velocity, thus resulting to a broader power harnessing range. On the other hand, the harnessed power at the end of the VIV upper branch is lower than that of the linear stiff spring $K=1,000 \mathrm{~N} / \mathrm{m}$ by about $40 \%$ due to its lower frequency response.

(b) $70,000 \leq R e \leq 80,000 \sim 87,000$ : This region is past the VIV upper branch to the VIV lower branch to desynchronization. The harnessed power drops sharply for all the nonlinear stiffness functions due to the drop in amplitude at the separation between VIV and galloping. These are anticipated based on the earlier discussion related to the equations (1) and (3).

(c) $87,000-94,000 \leq R e$ : This is the transition region between VIV and galloping, followed by the fully developed galloping region. The harnessed power increases as the flow velocity increases. Compared to the linear-spring stiffness system, the nonlinear-spring system shows a steeper power increase due to the softer $K_{l}$ spring, which allows larger galloping amplitudes and makes the cylinder bounce off the $K_{2}$ springs.

At the initial galloping stage for $87,000<R e<100,000$, the $y_{0} / D=2.5$ function has the highest harnessed power at $14.2 \mathrm{~W}\left(c_{\text {harness }}=30 \mathrm{Ns} / \mathrm{m}\right)$, compared to $y_{0} / D=2.0$ function at $13.2 \mathrm{~W}$, and $y_{0} / D=3.0$ function at $13.0 \mathrm{~W}$. Moving to fully developed galloping, $c_{\text {harness }}=30 \mathrm{Ns} / \mathrm{m}$ results in the 
highest harnessed power for all the nonlinear stiffness functions. Overall, $c_{\text {harness }}=30 \mathrm{Ns} / \mathrm{m}$ along with $y_{0} / D=2.5$ yields the highest harnessed power expect for $R e=118,000$.

Compared to both linear stiffness cases in Fig. 12, all the nonlinear restoring functions harness $76 \%$ more power at the beginning of galloping and throughout all the galloping range.

Data for Reynolds number greater than 120,000 is not available due to the flow velocity reaching the safety limits of the Channel. The upward trend, however, shows the potential of achieving high power.

\subsection{Conversion Efficiency}

The hydrokinetic power in a fluid flow through surface $\mathrm{S}$ is

$$
P_{\text {Fluid }}=\frac{1}{2} \rho U^{3} S
$$

In the case of an oscillating cylinder and for comparison to the area of a propeller disk, the area used is that swept by the cylinder. This yields

$$
P_{\text {Fluid }}=\frac{1}{2} \rho U^{3}\left(2 A_{\text {max }}+D\right) L
$$

where $A_{\max }$ is the largest amplitude among all cylinders in a multiple-cylinder converter. In this work where only one oscillator is available, only one $A_{\max }$ is defined. Obviously, the denominator depends on the cylinder response, unlike propellers or wind turbines where the flow area is determined by the fixed geometry of the area swept by the blades. The Betz limit is the theoretical maximum power that can be extracted from an open flow and is equal to $59.26 \%$ 
$(=16 / 27)$. Based on the Betz limit, the power conversion efficiency, $\eta_{\text {converted }}$, and power harnessing efficiency, $\eta_{\text {harnessed, }}$, can be calculated as

$$
\begin{aligned}
& \eta_{\text {converted }}(\%)=\frac{P_{\text {converted }}}{P_{\text {fluid }} \cdot(\text { Betz Limit })} \cdot 100 \\
& \eta_{\text {harnessed }}(\%)=\frac{P_{\text {converted }}}{P_{\text {fluid }} \cdot(\text { Betz Limit })} \cdot 100
\end{aligned}
$$

Commercial scale wind turbines achieve peak efficiency of $75 \%$ to $80 \%$ of the Betz limit (Barton et al. 2011). The two efficiencies modeled by equations (17) and (18) are plotted in Fig. 13 with the harnessing damping $c_{\text {harness }}$ as the parameter. The efficiency scales are shown on the right and left $y$-axis, respectively. Since, Fig. 12 shows that the overall best performance, occurred for the nonlinear restoring Function $2\left(y_{0} / D=2.5\right)$ only the corresponding efficiencies are plotted in Fig. 13. 


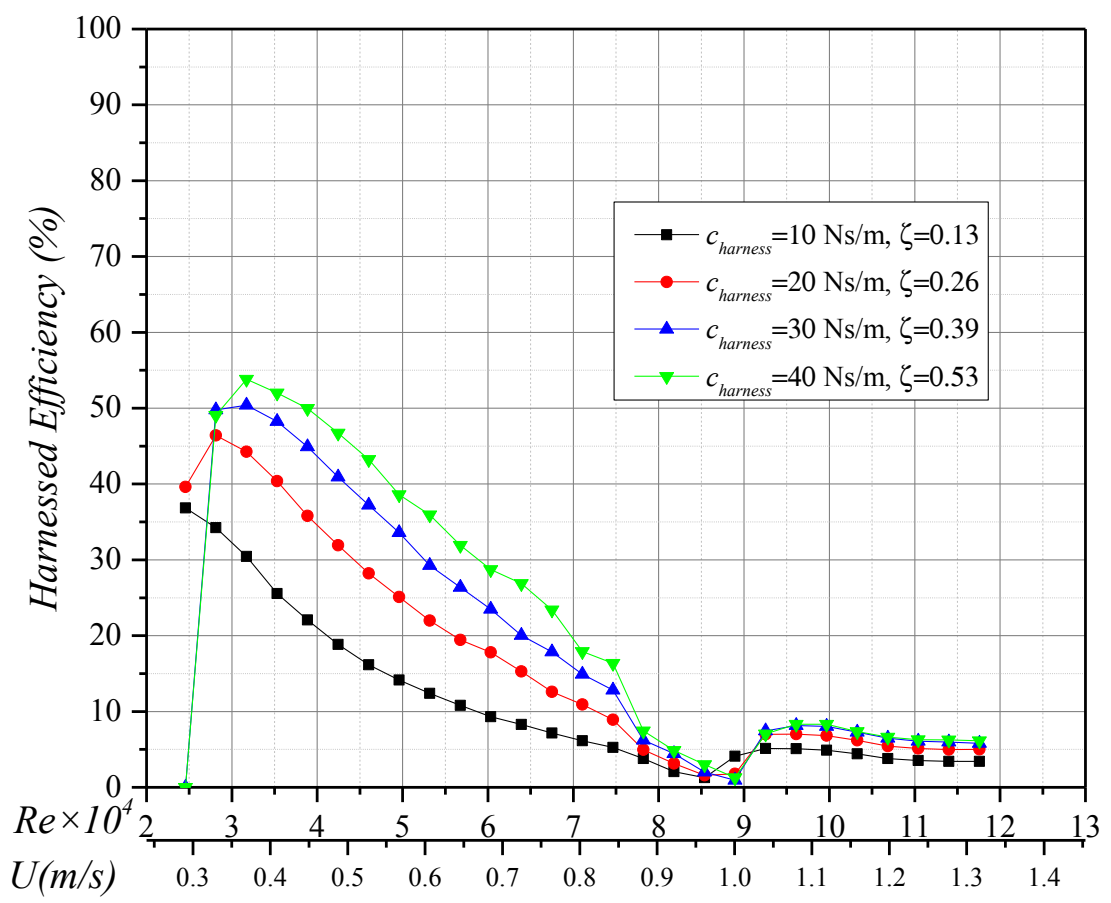

Figure 13. Harnessed and converted efficiency for nonlinear restoring force $k_{l}=200 \mathrm{~N} / \mathrm{m}$ and $k_{2}=1,000 \mathrm{~N} / \mathrm{m}$; four harness damping values $\mathrm{c}_{\text {harness }}$; the corresponding damping ratio to $K_{1}$ are $\zeta$ $=0.13,0.26,0.39,0.53$; mass ratio $m^{*}=1.343$; nonlinear piecewise function $y_{0} / D=2.5$.

Three distinctive regions can be observed:

(i) The VIV region, where efficiency exhibits a local optimum at the beginning of the upper branch for each harnessing damping, value. Specifically, the efficiency $\eta_{\text {harnessed }}$ for $c_{\text {harness }}=10 \mathrm{Ns} / \mathrm{m}$ reaches optimum of $36 \%$ of the Betz limit at $U=0.275 \mathrm{~m} / \mathrm{s}$ and then decreases until reaching the VIV to galloping transition region. The efficiency for $c_{\text {harness }}=40 \mathrm{Ns} / \mathrm{m}$ has the highest overall optimum ( $54 \%$ of Betz limit) at $U=0.35 \mathrm{~m} / \mathrm{s}$. The optimum of $c_{\text {harness }}=20 \mathrm{Ns} / \mathrm{m}$ and $c_{\text {harness }}=30 \mathrm{Ns} / \mathrm{m}$ are $46 \%$ and $50 \%$, respectively. 
Comparing Fig. 12 to Fig. 13 shows that the power local optimum and the efficiency local optimum in VIV do not coincide. The VIV power local optimum is at the end of the VIV upper branch, while the efficiency local optimum is at the beginning of the upper branch. The explanation can be found in equations (17) as $A_{\max }$ increases along the upper branch with increasing $U$ in the TrSL3 flow regime.

(ii) The VIV to galloping transition region, where the efficiency for $c_{\text {harness }}=40 \mathrm{Ns} / \mathrm{m}$ is higher than the rest of the damping values except at $R e=89,000$. This exception occurs because the initiation of galloping occurs earlier for lower harnessing damping $c_{\text {harness }}=10 \mathrm{Ns} / \mathrm{m}$ as equation (3) shows. The reason for low efficiency at the maximum overall power is due to the same reason that was stated previously; the denominator in equation (17) increases with $A_{\max }$ as defined by equation (16).

(iii) The galloping region, where the efficiency increases as the harness damping increases up to certain value. The efficiency curves in Fig.13 show that the local optimum is reached at the beginning of galloping and has a constant decrease as the flow velocity increases. Again this is due to the increase in $A_{\max }$ with increasing $R e$, and accordingly the area swapped by the cylinder and the denominator in equations (17) and (18).

In Fig. 13, the efficiency curves for $c_{\text {harness }}=30 \mathrm{Ns} / \mathrm{m}$ and $c_{\text {harness }}=40 \mathrm{Ns} / \mathrm{m}$ cross at several test points, which indicates that the optimal power output of the tested VIVACE Converter Vck system has been reached. When the harness damping was increased further, the converted power was reduced. For the same efficiency, however, the amplitude of $c_{\text {harness }}=30 \mathrm{Ns} / \mathrm{m}$ is higher than 
the amplitude for $c_{\text {harness }}=40 \mathrm{Ns} / \mathrm{m}$. That is, the former has the highest harnessed power for all the nonlinear spring stiffness tested.

In the galloping instability range, the efficiency local optimum is a boundary point indicating that its value increases as the flow velocity increases beyond the LTFSW Cannel capacity. The local optimum of power and local optimum of efficiency do not match and each one represents a very different design of the Converter. The efficiency local optimum is located at the beginning of the fully developed galloping, while the power local optimum is located at the end of the galloping range.

VIV is self-limiting yielding an interior design-space point as the optimum. Galloping, on the other hand, is an instability based phenomenon and the cylinder can move in one direction as long as the Converter frame allows it. In a very large frame, high power would be produced but a higher denominator would make the efficiency nearly zero.

\section{SUMMARY AND CONCLUSIONS}

The flow induced motions and the hydrokinetic power conversion capacity of a single, rigid, circular cylinder with PTC, in the form of selectively distributed local roughness, on nonlinear piecewise restoring force/spring for various values of functions and harnessing damping were investigated experimentally at Reynolds numbers in the TrSL3 flow regime $(24,000<\operatorname{Re}<120,000,0.274 \mathrm{~m} / \mathrm{s}<U<1.315 \mathrm{~m} / \mathrm{s})$. Three nonlinear restoring spring/force models

were tested with two segments; an initial soft one followed by a short stiffer spring intended to 
reverse the motion direction efficiently. The location of the transition point between the softer and stiffer springs was changed between the three models.

All experiments were conducted in the LTFSW Channel of the MRELab of the University of Michigan using the second generation of the Vck virtual oscillator developed in the MRELab. The results, including amplitude response, frequency response, energy conversion, and energy conversion efficiency of the single-cylinder Converter as function of Reynolds number and flow velocity were presented and analyzed.

There are differences and similarities between the nonlinear systems tested in this work and linear systems tested in previous work (Chang et al. 2011, Park et al. 2013b). The major differences are the following:

(1) Due to the soft first segment in the nonlinear restoring force, power harnessing was initiated at a lower flow velocity $(0.275 \mathrm{~m} / \mathrm{s}, R e \leq 25,000,0.53$ knots $)$ than previous tests with linear restoring force.

(2) In galloping, power harnessing is higher with the nonlinear restoring force than the corresponding case with linear restoring force. At the onset of galloping the difference is about $76 \%$. As the flow velocity increases this difference reduces to about $15 \%$.

(3) The values of harnessing damping tested were sufficient to reveal the optimal power for the nonlinear stiffness model. Specifically, the highest harnessed power was generated at the highest velocity tested $(1.32 \mathrm{~m} / \mathrm{s})$, at $c_{\text {harness }}=30 \mathrm{Ns} / \mathrm{m}$, for all three nonlinear stiffness functions: $17.55 \mathrm{~W}$ for Function $1\left(y_{0} / D=2.0\right), 17.44 \mathrm{~W}$ for Function $2\left(y_{0} / D=2.5\right)$, and 16.71 
$\mathrm{W}$ for Function $3\left(y_{0} / D=3.0 \mathrm{D}\right)$. Higher damping for energy harnessing would result in a larger gap between the VIV synchronization range and the onset of galloping, thus having a negative impact on the range covered by Converter oscillators.

(4) In galloping when reaching the $K_{2}$ segment stiffness, there is still one predominant frequency of oscillation, which increases as the flow velocity increases, contrary to the linear restoring force case.

The following similarities between the nonlinear systems tested in this work and linear systems tested in previous work were observed:

(5) Regardless of whether the oscillator motion is in the galloping or the VIV region, there is only one predominant frequency.

(6) Power curves exhibit two local optima, one in the VIV range and one in the galloping range. The VIV local power optimum is located at the end of the upper branch at $c_{\text {harness }}=40 \mathrm{Ns} / \mathrm{m}$. The galloping local power optimum is located at the highest velocity achieved within the capabilities of the MRELab recirculating Channel at all harness damping values.

(7) Efficiency curves exhibit two local optima, one in the VIV range and one in the galloping range. The efficiency local optima do not coincide with the power local optima because the denominator in the efficiency is a function of the amplitude of oscillation and flow velocity.

(8) The efficiency local optimum in VIV is located at the beginning of the upper branch, which represents the lowest velocity after the initial branch. This is associated with a high jump in amplitude but not a big drop in frequency resulting in a high increase in the numerator of 
equations (17) and (18). This increase exceeds the increase in equation (16) which represents the denominator of the efficiency.

(9) The galloping local optimum of the efficiency is located at the beginning of the fully developed galloping region, that is at the opposite end of the power optimum in the galloping region. In the galloping region, the efficiency decreases slowly after its peak.

\section{ACKNOWLEDGEMENTS}

Prepared under Cooperative Agreement No. DE-EE0006780 between Vortex Hydro Energy, Inc. and the U.S. Department of Energy. The MRELab is a subcontractor through the University of Michigan.

Prepared as an account of work sponsored by an agency of the US Government. Neither the US Government nor any agency thereof, nor any of their employees, makes any warranty, express or implied, or assumes any legal liability or responsibility for the accuracy, completeness, or usefulness of any information, apparatus, product, or process disclosed, or represents that its use would not infringe privately owned rights. Reference herein to any specific commercial product, process, or service by trade name, trademark, manufacturer, or otherwise does not necessarily constitute or imply its endorsement, recommendation, or favoring by the US Government or any agency thereof. The views and opinions of authors expressed herein do not necessarily state or reflect those of the US Government or any agency thereof.

\section{REFERENCES}

[1] AR Quadrante, L. and Nishi Y., (2013), "Attachment of Tripping Wires to Enhance the Efficiency of a Vortex-Induced Vibrations Energy Generation System." Journal of Power and Energy Systems 7.3: 162-176.

[2] Barrero-Gil, Antonio, Santiago Pindado, and Sergio Avila., (2012), "Extracting energy from vortex-induced vibrations: a parametric study." Applied Mathematical Modeling 36.7: 3153-3160. 
[3] Barton, David AW, Stephen G. Burrow, and Lindsay R. Clare, (2010), "Energy harvesting from vibrations with a nonlinear oscillator." Journal of Vibration and Acoustics 132.2, 021009 .

[4] Bearman, P.W., (2011), "Circular cylinder wakes and vortex-induced vibrations”, Journal of Fluid Mechanics 27, 648-658.

[5] Bernitsas M.M., Raghavan K., (2009), "Converter of Current, Tide, or Wave Energy", United States Patent and Trademark Office, Patent\# 7,493,759 B2 issued on February 24.

[6] Bernitsas, M. M., Raghavan,K., (2011), "Enhancement of Vortex Induced Forces \& Motion through Surface Roughness Control" US Patent Trademark Office, Pat.\# 8,047,232 B2, Nov. 1.

[7] Bernitsas, M. M., (2016), "Harvesting Energy by Flow Included Motions", Chapter 47, Springer Handbook of Ocean Engineering, Editors: Dhanak, M. R., Xiros, N. I., Springer-Verlag Berlin Heidelberg.

[8] Blevins, R. D., (1990), Flow-Induced Vibration, $2^{\text {nd }}$ Edition, Van Nostrand Reinhold, New York, USA.

[9] Chang, C.C., Kumar, R.A., Bernitsas, M.M., (2011), "VIV and galloping of single circular cylinder with surface roughness at $3.0 \times 10^{4} \leq \mathrm{Re} \leq 1.2 \times 10^{5}$." Journal of Ocean Engineering 38.16, 1713-1732.

[10] Chomaz, J. M., Philippe Meliga, and Francois Gallaire, (2011), "Optimizing energy production from vortex-induced vibrations using control strategy deduced from an exact nonlinear asymptotic model." 6th AIAA Theoretical Fluid Mechanics Conference.

[11] Kinaci, O. K., M.M. Bernitsas, Sami Lakka, Hai Sun, (2016), "Effect of Tip-Flow on Vortex Induced Vibration of Circular Cylinders for Re $\leq 1.2 \times 105$ ”, Journal of Ocean Engineering, in press.

[12] Lee, J.H., Xiros, N. and Bernitsas, M.M., (2011), "Virtual damper-spring system for VIV experiments and hydrokinetic energy conversion." Ocean Engineering 38.5 , 732-747.

[13] Lee, J.H., and Bernitsas, M.M., (2011), "High-damping, high-Reynolds VIV tests for energy harnessing using the VIVACE converter." Ocean Engineering 38.16, 1697-1712. 
[14] Meliga, P. and Chomaz, J., (2011), "An asymptotic expansion for the vortex-induced vibrations of a circular cylinder." Journal of Fluid Mechanics 671, 137-167.

[15] Mackowski, A. W., and C. H. K. Williamson, (2013), "An experimental investigation of vortex-induced vibration with nonlinear restoring forces." Physics of Fluids (1994-present) 25.8, 087101.

[16] Park, H. R., Bernitsas, M. M., Chang, C.C., (2013a), "Robustness of the Map of Passive Turbulence Control to Flow-Induced Motions for a Circular Cylinder at $30,000<\operatorname{Re}<120,000$ ", Proceedings of the 31st OMAE 2013 Conf., Paper \#10123, Nantes, France, June 9-14, 2013.

[17] Park, H. R., R. A. Kumar, Bernitsas, M. M., (2013b), "Enhancement of Flow Induced Motions of Rigid Circular Cylinder on Springs by Localized Surface Roughness at $3 \times 10^{4} \leq \operatorname{Re} \leq 1.2 \times 10^{5 "}$, Ocean Engineering, Vol. 72, 1, Pages 403-415.

[18] Raghavan, K., Bernitsas, M.M., (2011), "Experimental Investigation of Reynolds Number Effect on Vortex Induced Vibration of Rigid Cylinder on Elastic Supports," Ocean Engineering, Vol.38, \#5-6, pp.719-731.

[19] Sarpkaya, T., (2004), "A critical review of the intrinsic nature of vortex-induced vibrations." Journal of Fluids and Structures 19.4, 389-447.

[20] Smogeli, Ø. N., Hover, F. S., and M. S. Triantafyllou, (2003), "Force-feedback control in VIV experiments," in Proceedings of the OMAE 22nd International Conference on Offshore Mechanics and Arctic Engineering, ASME Transactions, Cancun, Mexico, p. 685.

[21] Sun, H. Kim, E. S., Bernitsas P. M, Bernitsas M.M., (2015), "Virtual Spring-Damping System for Flow-Induced Motion Experiments," Journal of Offshore Mechanics and Arctic Engineering 137.6, 061801.

[22] Sun, H. Kim, E. S., Nowakowski, G, Mauer, E. Bernitsas, M. M. Effect of mass-ratio, damping, and stiffness on optimal hydrokinetic energy conversion of a single, rough cylinder in flow induced motions Renewable Energy. 10.1016/j.renene.2016.07.024 
[23] Walker, D. T., Lyzenga, D. R., Ericson, E. A., \& Lund, D. E. (1996). Radar backscatter and surface roughness measurements for stationary breaking waves. In Proceedings of the Royal Society of London A: Mathematical, Physical and Engineering Sciences (Vol. 452, No. 1952, pp. 1953-1984). The Royal Society.

[24] Zdravkovich, M. M., (1997), "Flow Around Circular Cylinders", Vol. 1, E. Achenbach, ed., Oxford University Press, Oxford, UK. 


\section{NOMENCLATURE}

Greek symbols:

\begin{tabular}{|l|l|}
\hline$\alpha_{P T C}$ & Strip placement angle \\
\hline$\theta$ & Angle coverage of the passive turbulence control \\
\hline$\zeta_{\text {structure }}$ & Damping ratio due to losses in the transmission system \\
\hline$\zeta_{\text {harness }}$ & Harness damping ratio \\
\hline$\zeta_{\text {converted }}$ & Converted damping ratio \\
\hline$\omega_{\text {osc }}$ & Angular frequency of oscillation \\
\hline$\rho$ & Water density \\
\hline$\mu$ & Dynamic Viscosity of water \\
\hline$v$ & Kinematic Viscosity of water \\
\hline
\end{tabular}

Roman symbols:

\begin{tabular}{|l|l|}
\hline$A$ & Amplitude value of the transverse displacement \\
\hline$A^{*}$ & Amplitude ratio $A / D$, average of the 60 highest peaks \\
\hline$c$ & Total damping \\
\hline$c_{\text {harness }}$ & Harnessing damping \\
\hline$c_{\text {virtual }}$ & Added linear damping from Vck system \\
\hline$D$ & Diameter of cylinder \\
\hline$f_{n, \text { water }}$ & Natural frequency in water \\
\hline$f_{\text {osc }}$ & Oscillation frequency of cylinder \\
\hline$f^{*}$ & Oscillation frequency ratio in water \\
\hline$F_{\text {fluid }}$ & Force exerted by the fluid on the cylinder in the $y$-direction \\
\hline$F_{\text {nonlinear_damping }}$ & $\begin{array}{l}\text { Nonlinear damping from the system, containing the friction from structure and Vck } \\
\text { system }\end{array}$ \\
\hline$F_{\text {controller }}$ & Force applied by the Virtual system (passive) \\
\hline$K$ & Spring stiffness \\
\hline$L$ & Cylinder length \\
\hline$m_{\text {dis }}$ & Displacement mass \\
\hline$m_{\text {osc }}$ & Oscillating mass \\
\hline$m_{a}$ & Added mass \\
\hline$m^{*}$ & Mass ratio \\
\hline$P_{\text {harnessed }}$ & Harnessed hydrokinetic power from the flow \\
\hline$P_{\text {converted }}$ & Total hydrokinetic power from the flow \\
\hline$P_{\text {dissipated }}$ & Dissipated hydrokinetic power due to structural damping \\
\hline$R e$ & Reynolds number \\
\hline
\end{tabular}




\begin{tabular}{|l|l|}
\hline$t$ & Time \\
\hline$T_{\text {osc }}$ & Oscillation period \\
\hline$U^{*}$ & Reduced velocity \\
\hline$y$ & Instantaneous cylinder motion in transvers direction \\
\hline$\dot{y}$ & Instantaneous cylinder velocity in transvers direction \\
\hline$\ddot{y}$ & Instantaneous cylinder acceleration in transvers direction \\
\hline
\end{tabular}

